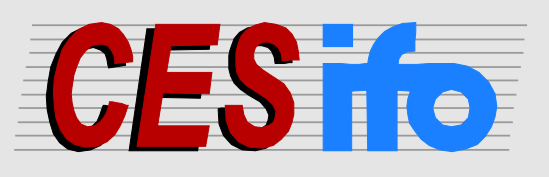

\title{
Working
}

Papers

www.cesifo.org/wp

\section{Tailored Bayesian Mechanisms: Experimental Evidence from Two-Stage Voting Games}

\author{
Dirk Engelmann \\ Hans Peter Grüner
}

CESIFO WORKING PAPER NO. 6405

CATEGORY 13: BEHAVIOURAL ECONOMICS

MARCH 2017

An electronic version of the paper may be downloaded

- from the SSRN website:

- from the RePEc website:

- from the CESifo website:

www.SSRN.com

www.RePEc.org

www.CESifo-group.org/wp

ISSN 2364-1428

\section{CESifo}




\title{
Tailored Bayesian Mechanisms: Experimental Evidence from Two-Stage Voting Games
}

\begin{abstract}
Optimal voting rules have to be tailored to the underlying distribution of preferences. This paper shows that the introduction of a stage at which agents may themselves choose voting rules according to which they decide in a second stage may increase the sum of individuals' payoffs if players are not all completely selfish. Our experiments aim to understand how privately informed individuals choose voting rules and vote given these rules. In a setting with an asymmetric distribution of valuations groups that can choose a voting rule do better than those who decide with a given simple majority voting rule.
\end{abstract}

JEL-Codes: C910, D700, D820.

Keywords: two-stage voting, Bayesian voting, experiments.

\author{
Dirk Engelmann \\ Humboldt-University Berlin \\ Department of Economics \\ Spandauer Str. 1 \\ Germany - 10099 Berlin \\ dirk.engelmann@hu-berlin.de
}

\author{
Hans Peter Grüner \\ University of Mannheim \\ Department of Economics, L7, 3-5 \\ Germany - 68131 Mannheim \\ gruener@uni-mannheim.de
}

March 13, 2017

We thank Jana Friedrichsen, Sander Renes, Thomas Tröger and Philipp Zahn as well as seminar participants at WZB Berlin, DICE Düsseldorf, Tinbergen Institute Amsterdam, Royal Holloway, MaxPlanck Institute Bonn, CERGE-EI Prague, HECER Helsinki, NHH Bergen, the Universities of Zürich, Mannheim, Utrecht, Tilburg, Rotterdam, Erlangen-Nürnberg, Konstanz, Edinburgh, Maastricht, Mainz, and British Columbia, the Schumpeter seminar at HU and FU Berlin, and participants at the International ESA Conference in New York 2012, the ESA European Conference in Cologne 2012, the 13th SAET International Conference on Current Trends in Economics in Paris 2013, the 2013 meeting of the economic theory group of the German Economic Association, the 2014 annual meeting of the Verein für Socialpolitik in Hamburg, and the 2014 CESifo Area Conference in Behavioural Economics in Munich for their useful comments. We also thank Johannes Bubeck and Christoph Siemroth for excellent research assistance. Financial support from the German research foundation (DFG), SFB 884 is gratefully acknowledged. 


\section{Introduction}

Many collective decisions are governed by rules. Ideally, these rules should be designed to fit to the underlying problem - but very little is known about what motivates people when they select them. This paper studies the choice of decision making rules with a series of laboratory experiments. Our experiments focus on the choice of voting rules by individuals whose interests have already materialized. At this stage it is particularly difficult to efficiently tailor rules because the choice and acceptance of rules may be governed by individuals' selfish preferences over outcomes rather than their concern for fair or efficient procedures. Instead, we find that the procedural choices of a substantial share of subjects is in line with altruistic motivations. Specifically, when preferences are distributed symmetrically, many voters are willing to use efficient symmetric rules even when they would themselves benefit from a rule that is biased in favor of their preferred alternative. In asymmetric settings, a substantial share of voters is willing to protect minority interests with a supermajority rule. Consequently, procedures that allow for informed collective decisions about rules may be welfare enhancing.

It is a key politico-economic insight that rules governing collective decision making should ideally be determined before individual preferences about outcomes have realized. Consensual and efficient choices are easier to obtain on the constitutional level because individuals cannot be sure about their situation and preferences in the future (Brennan and Buchanan, 1985). ${ }^{1}$ While convincing in theory, the concept of a choice of decision making rules, ignorant of own preferences, has limited applicability in practise beyond broadly applicable rules. First, new upcoming collective decision problems may require tailor-made decision rules - such as voting or procedural rules - that cannot be fixed in a constitution beforehand. Second, decision problems often appear simultaneously with private knowledge about individual preferences over possible outcomes. As a result, by the time adjustments to the decision rule have to be made that take specifics of the decision problem at hand into account, these adjustments cannot be made ignorant of own preferences anymore. Therefore, it is important to understand, whether rules can be tailored to major features of the underlying problem at the interim stage, when preferences over outcomes have already realized.

Indeed, interim choices of rules are far more widespread in practice than one would

\footnotetext{
${ }^{1}$ The applicability of rules across numerous decision problems and their validity for longer time horizons is crucial for agreements on efficient rules being easier than agreements on efficient outcomes. Along similarly lines, Rawls (1972) argued that distributional rules should ideally correspond to the ones that an individual uninformed about his own position in society would pick.
} 
believe based on theoretical reasoning about their usefulness. One important political body that fixes its own rules at the interim stage is the U.S. Congress. According to article I, Section 5 of the U.S. constitution, "Each House may determine the Rules of Its Proceedings". This is done on a case by case basis, which usually implies that preferences of the members of congress have at least to some extent already realized. While these rules choices are mostly procedural (see Oleszek, 2014, for a detailed overview), they may still have a major influence on the chances of a particular outcome. The power of the rules committee has been confirmed by the former House minority leader Robert H. Michel, who argued that "Procedure hasn't simply become more important than substance - it has (...) become the substance of our deliberations. Who rules House procedures rules the House (...)" (quote from Oleszek, 2014, p.12). Also beyond the U.S. Congress it is not uncommon for committees to first decide about procedural rules or voting thresholds before debating and deciding about the issue itself. These rules often take information about the underlying distribution of preferences at least implicitly into account. $^{2}$

Our empirical analysis concentrates on the choice among a practically relevant class of decsion making mechanisms - voting rules. A major problem related to the construction of voting mechanisms is that the threshold for a majority vote should ideally be tailored to the underlying distribution of preferences. Several theoretical papers address how optimal majority rules have to be adjusted when the underlying distribution of preferences changes, ${ }^{3}$ but very little is known about how this flexibility could be achieved in practice.

There are several reasons why it is difficult in practice to properly adjust the voting rule to the underlying problem. One theoretical option would be to design a "complete" constitution that lists all possible decision problems. Obviously, this option is far too costly. Many constitutions very broadly distinguish only a few categories of decision problems and allocate different majority thresholds to these categories (for example,

\footnotetext{
${ }^{2}$ Another example of rule choice at the interim stage is the United Nations General Assembly which occasionally chooses to proceed under consensus when the issue is particularly controversial (http://www.nationsencyclopedia.com/United-Nations/Comparison-with-the-Leagueof-Nations-VOTING.html).

${ }^{3}$ Examples are Ledyard and Palfrey (2002), who study asymptotically optimal referenda and Schmitz and Tröger (2012) who derive a condition for the optimality of the simple majority rule. A literature that follows Austen-Smith and Banks (1996) and Feddersen and Pesendorfer (1998) studies the effects of various voting thresholds in a jury setup with identical preferences and heterogeneous information. Guarnaschelli, McKelvey, and Palfrey (2000) find that individual voting behavior reacts to the majority threshold qualitatively in line with the theoretical predictions.
} 
changes of the constitution itself often require a two-thirds majority). Another theoretical option is to let some benevolent planner who knows the underlying distribution of preferences adjust the voting rules to that distribution. This option is unrealistic in practice because perfectly benevolent individuals may be rare and, even if they exist, hard to identify. Furthermore, their political legitimacy for enforcing such a rule may be questioned.

Instead, one may consider to let voters themselves decide on the appropriate majority threshold whenever a new issue comes up. In theory, this can properly solve the problem of selecting an optimal mechanism if this selection takes place at the ex-ante stage where individuals do not yet know their own preferences but know the underlying distribution. However, it is not straightforward that a rule-choice stage at a point when agents are already informed about their preferences increases welfare.

This paper studies whether the introduction of a stage at which agents choose the rules according to which they decide may help to solve the problem that voting inefficiently aggregates preferences and whether adding a rule-choice stage increases the sum of individuals' payoffs. We conduct an experiment with three closely related treatments to understand how individuals decide when they choose voting rules at the interim stage. Specifically, we consider an environment in which agents can vote on a status quo with predetermined identical payoffs (alternative B) and an alternative with stochastic and often unequal payoffs (alternative A). The difference between both payoffs is hereafter called an agent's valuation of A. Agents privately learn about their randomly chosen valuation in the first stage. Then they decide on the voting rule and are informed about the implemented rule before voting takes place. In our setup, introducing a procedural choice stage is useless if agents act selfishly because they should pick the rule that makes their preferred outcome most likely to obtain. ${ }^{4,5}$ However, our paper shows theoretically that if subjects exhibit efficiency concerns, ${ }^{6}$ then the interim rule-choice stage is not

\footnotetext{
${ }^{4}$ More precisely, this holds if all are selfish and this is common knowledge. Otherwise, it is at least conceivable that even if all are selfish, but do not expect all others to be selfish, they could try to use a non-selfish vote at the rule choice stage to signal something about their valuation which might trigger non-selfish voting behavior in others, from which they would in turn benefit. However, at least for the specific rule choice procedures that we study in our first two treatments, this is not a concern, because whenever one's own rule choice becomes relevant and known, one can unilaterally determine the outcome and there is thus no incentive for selfish players to try to signal a different type.

${ }^{5}$ Trebbi, Aghion, and Alesina (2008) provide evidence that the choice of electoral rules is indeed motivated egoistically.

${ }^{6}$ In line with the literature (Charness and Rabin, 2002, Engelmann and Strobel, 2004) we use the term "efficiency concerned" for subjects who care about the - possibly weighted - sum of individual
} 
redundant. Our experiments study to what extent efficiency-concerned subjects choose welfare-enhancing voting rules, which fit to the underlying distribution of preferences.

The first main finding of our experiments is that agents often do not act selfishly at the rule-choice stage, but instead propose rules which are biased towards the efficient (sumof-payoffs maximizing) rule. We study this effect in two different treatments. Our first treatment with a symmetric and fine distribution of valuations serves as an initial test to what degree participants deviate from selfish rules and selfish voting. Our second treatment with a strongly asymmetric distribution of valuations permits to investigate the possibility of obtaining efficiency gains through the endogenous determination of voting thresholds. With an asymmetric distribution, the efficient majority threshold changes and efficiency-oriented subjects' deviations from proposing the selfish rule adjust accordingly. Indeed, we find evidence suggestive of efficiency concerns at the rule-choice stage. Welfare is slightly higher than in the third treatment that uses the same distribution of preferences but pre-determined majority voting.

As a second main finding, results from our first two treatments suggest that some agents follow different motivations at the rule-choice stage than at the voting stage. Specifically, their behavior at the rule-choice stage seems to be more efficiency driven than at the voting stage. At the rule-choice stage we find substantial evidence for efficiency concerns and no evidence for inequality aversion, a social preference that has been argued to matter in many experiments (Bolton and Ockenfels, 2000, Fehr and Schmidt, 1999), whereas at the voting stage, behavior looks mostly selfish and only occasionally in line with inequality aversion or efficiency concerns. Hence adding a rulechoice stage can potentially increase efficiency because efficiency concerns may be more important on the rule-choice stage, apparently because choosing a rule suggests that one should do the right (i.e., efficient) thing.

Any given voting mechanism can only imperfectly aggregate the available information about individuals' valuation of the relevant alternatives. Therefore, it may occur that many agents with a small positive valuation for some decision trigger a decision which creates much larger losses for a small number of agents. Introducing a rule-choice stage in principle permits subjects to send finer signals about their valuation at that stage if the available set of rule choices is sufficiently rich. In theory, they should not make use of this option and choose extreme voting rules whenever valuations differ from zero.

payoffs. Efficiency concerns in this sense should not be equated with a concern for Pareto-optimality. Obviously, in a setup with positive and negative valuations, all collective decisions are Pareto-optimal if one does not permit monetary transfers. 
We find that, in practice, a sizable share of subjects suggest voting rules that do react gradually to their valuation. ${ }^{7}$

The question how to tailor mechanisms to the underlying distribution of types is of more general relevance and has been addressed in the literature on optimal auction design (Myerson 1981) as well as on optimal compromising (Börgers and Postl, 2009). ${ }^{8}$ While adjusting the mechanism to the preference distribution seems to be a feasible task in theory, it may be difficult to find an appropriately motivated person to perform this task in many practical applications. ${ }^{9}$ Our experimental results indicate that efficiencyconcerned agents may actually be willing and able to perform this task.

The major part of the mechanism design literature concentrates on the design of normal form games. We show that adding a theoretically irrelevant procedural-choice stage affects outcomes and therefore the restriction to one-stage games is problematic. ${ }^{10}$ In our conclusion we discuss how this effect could be exploited in the design of collective decision mechanisms.

Our paper is related to a literature that studies the optimal design of Bayesian voting mechanisms (e.g., Schmitz and Tröger, 2012). It also contributes to a - so far mostly theoretical - literature that studies the acceptance of collective decision making mechanisms at the interim stage that started with the seminal paper by Myerson and Satterthwaite (1983). ${ }^{11}$ In another related theoretical paper, Barbera and Jackson

\footnotetext{
${ }^{7}$ Our experimental design does not fully exploit the possible efficiency gains from gathering finer signals about players' preferences. We implement the rule choice through a random-dictator mechanism. Each player in a group suggests a threshold for the second-stage vote and then one of them is randomly chosen and this rule is implemented in the second stage. This allows a player whose rule is chosen to unilaterally implement his preferred outcome. If he prefers A, he picks as the rule that unanimity is required for B and votes for A and vice versa if he prefers B. The reason we choose this mechanism in the experiment is that as a first step we wanted to understand participants' preference over rules and this mechanism allows to elicit these in a transparently incentive compatible way. A rule-choice mechanism that makes better use of the entire information gathered in this stage than the random-dictator mechanism could potentially further improve welfare.

${ }^{8} \mathrm{~A}$ related question considers the optimal choice of a mechanism by an informed principal, who does not want to reveal information through his choice of mechanism (Myerson 1983). In our case, revealing private information is instead potentially beneficial if other voters are efficiency concerned.

${ }^{9}$ The difficulties that are associated with the task to tailor mechanisms to the distribution of players' types are one reason why a substantial part of the mechanism design literature focuses on different forms of robust implementation (Wilson, 1985, Bergemann and Morris, 2005, 2012, Bierbrauer and Hellwig, forthcoming).

${ }^{10}$ In a recent paper, Masatlioglu, Taylor und Uler (2012) show experimentally that different one-stage auction games, which in theory produce identical equilibrium social choice functions lead to different bidding behavior in the laboratory.

${ }^{11}$ Further contributions on interim participation decisions in the context of other collective choice
} 
(2004) study the dynamic stability of pairs of voting rules (for constitutions and single issues) when single-issue rules can be changed over time.

A recent theoretical and experimental literature studies conflict resolution under private information about preferences when several related decisions can be bundled (e.g., Casella, 2005, Casella, Gelman, and Palfrey., 2006, Jackson and Sonnenschein, 2007, Hortala-Vallve and Llorente-Saguer, 2010, Engelmann and Grimm, 2012, Kaplan and Ruffle, 2012). Bundling makes it possible to provide individuals with incentives to adjust their messages about preference intensity gradually to their private information. It thus allows for efficient conflict resolution without adding a second decision stage. Our experiment focuses on decision problems that cannot be bundled.

There is an empirical literature that studies preferences for electoral rules based on survey questions, quasi-experiments or single voting outcomes (see Blais et al., 2015, and the references therein). Instead, our paper tests the choice of mechanisms at the interim stage in a controlled and incentivized experiment. A more recent paper by Weber (2016) experimentally studies the choice of voting rules for representatives of homogenous groups, but does so in a setup with binary valuations of identical absolute size that does not permit to study how the absolute size and size-distribution of individual valuations affect the choice of voting rules.

Another related experimental paper is Balafoutas et al. (2013) who investigate voting about the intensity of redistribution in a subsequent public-good game. Similar to our game, voting happens at the interim stage where participants already know which income group they are in, but in contrast to our experiment subjects do not choose the voting mechanism. ${ }^{12}$

Finally, while our experiment is neither intended to, nor very well suited to test participants' social preferences, we nevertheless gain some insights into the debate on the relative role of inequity aversion and concerns for payoff maximization (e.g., Charness and Rabin, 2002). Apart from the observation that we find evidence rather for efficiency concerns than for inequality aversion, one of our main results is that efficiency concerns may play a different role on different stages of a sequential game. Specifically, they appear to play a larger role on a rule-choice stage.

problems include Güth and Hellwig (1986), Cramton, Gibbons, and Klemperer (1987), Schmitz (2002), Segal and Whinston (2011), and Grüner and Koriyama (2012).

${ }^{12} \mathrm{~A}$ related experimental literature deals with the endogenous choice of sanctioning mechanisms in public good games, e.g., Kosfeld, Okada, and Riedl (2009). However, these experiments are typically based on symmetric games and thus heterogeneity of preferences is not induced and thus not controlled. 


\section{Experimental Design}

In this section, we first describe the underlying games and derive predictions for different types of preferences for our two main treatments and a control treatment with predetermined majority voting. Then we report the procedural details for these treatments.

\subsection{Treatment 1: Symmetric Valuation Distribution}

Our first treatment is designed to study whether behavior in the two-step procedure differs from rational maximization of one's own payoff and more specifically, to assess whether concerns for efficiency maximization are non-negligible compared to other motivations such as selfishness or inequality aversion in our two-step choice procedure. Only if there are non-trivial concerns for efficiency maximization is there a realistic perspective of two-step voting procedures to improve welfare. In order to have a chance to detect weaker as well as stronger degrees of efficiency concerns, we choose a valuation distribution with rather large and very small valuations, so that large benefits to others could come at various costs to one-self.

\subsubsection{The Game}

There are five players who collectively choose between two options, $A$ and $B$. If they choose option $B$, all players receive a payoff of 0 . If they choose option $A$, each player $i$ receives a payoff $\theta_{i}$ (Euro in the experiment). At the beginning of the game, the valuations $\theta_{i}$ are drawn from an i.i.d. distribution on a given set of values. The distribution of types is common knowledge and uniform on the set $\{-7,-3,-1,-0.5,-0.2,-0.1,0$, $0.1,0.2,0.5,1,3,7\}$. Each player only learns his own valuation $\theta_{i}$. Next, players decide between options $A$ and $B$ in a two-stage voting process. In the first of these stages each player proposes a voting rule from the set $\{1, \ldots, 5\}$. If rule $k$ is chosen, then in the second stage, $k$ votes are required to implement option $A$.

Subjects are informed that the actual rule is chosen by a random dictator mechanism, i.e., it is randomly chosen from the five voting rules that have been proposed by the five players. In the second stage of the two-stage voting process, players are informed about the chosen rule. They are not informed about the actual distribution of proposals or about the identity of the person whose proposal has been successful.

After being informed about the chosen voting rule, players vote about the two options. Either $A$ or $B$ is implemented depending on the rule chosen in the first stage and the 
votes from the second stage. If rule $k$ is chosen and at least $k$ players vote for $A$, then $A$ is implemented and each player $i$ receives $\theta_{i}$. If fewer than $k$ players vote for $A$, then $B$ is implemented and all players receive payoff 0 .

Players are informed about their own $\theta_{i}$ before the first stage but not about the complete vector of valuations. Note in particular that the choice of rules does thus not happen behind a veil of ignorance. Rules are thus to be understood here as ad-hoc rules for individual issues rather than as rules that are applied for a whole set of issues. Players are informed after each period of the game about the realized distributions of valuations in their group. Focusing on subjects' actions (and not counting the two random moves), we will henceforth call the rule proposal stage the first stage and the voting stage the second stage of the game.

\subsubsection{Theoretical Predictions}

Selfish Subjects Selfish players have weakly dominant strategies. In those strategies, they must choose rule 1 (5) if they have positive (negative) valuations, and, in the second stage, vote for their preferred outcome if they have non-zero valuations. Voters can choose any rule/outcome if their valuation is zero, and hence the dominant strategy is not unique. All other strategies are weakly dominated by those strategies because independent of the strategies of the other players, these strategies maximize the probability for outcome $A$ for $\theta>0$ and maximize the probability for outcome $\mathrm{B}$ if $\theta<0$.

Efficiency-concerned Subjects According to Schmitz and Tröger (2012), with a symmetric distribution of types, the simple majority rule maximizes the expected sum of individual payoffs. Suppose for a moment that all voters can be expected to vote "selfishly" in the second stage (we address below the case that others do not vote selfishly in the second stage). Consider players who do not simply want to maximize their own payoffs, but also care about the total payoff. In Appendix B we assume that subjects maximize a convex combination of all agents' monetary payoffs (with a larger weight on their own monetary payoff) and show that when they expect selfish play in stage 2 , they may propose rules $1,2,3,4$, or 5 , depending on the value of $\theta_{i}$. The proposed rule varies monotonically with the valuation. We note that for the symmetric distribution of valuations in Treatment 1 , the deviation from selfish rule choices is symmetric around a valuation of 0 and that the suggested rule will always be between the selfish rule and majority rule. That is, if $i$ has efficiency concerns and $\theta_{i}<0, i$ may choose rules 3,4 , or 5 , but not rules 1 or 2 and if $\theta_{i}>0, i$ may choose rules 1,2 , or 3 , but not rules 4 or 5 . 
There are a number of alternative assumptions that one could make regarding the subjects' expectations about the voting behavior of others. First, subjects could have rational expectations. Given that subjects only observe actual votes of other participants, but not the relation between individual valuations and votes it is rather implausible that they have rational expectations regarding the others' voting strategies. Furthermore, actual voting behavior is very close to selfish voting, so for the actual behavior that we observe replacing the assumption that subjects expect selfish voting with the assumption of rational expectations regarding the other participants' voting behavior makes very little difference. ${ }^{13}$

As a second alternative assumption, players could be subject to a so-called "false" consensus effect (Ross, Greene, and House, 1977, Engelmann and Strobel, 2000) and have beliefs that are biased towards their own type, in the extreme case believing that others behave exactly as they do. Assuming that others are equally efficiency concerned leads to complications if efficiency concerns are relatively strong. In this case, it could happen that a player proposes a different rule than under the assumption that all vote selfishly and then in the second stage votes against her preferences if she takes into account that choosing a different rule will change the voting behavior of others. As a result, the proposed rules may not change monotonically in the valuation. However, in the experiment we observe essentially no evidence of strategic voting, i.e., conditioning of the vote on the chosen rule, and therefore it does not appear to be a realistic assumption that players expect others to vote strategically.

\section{Preferences for "Democracy" and Selfishness with Error We note that in Treat-} ment 1 one cannot perfectly distinguish efficiency-concerned subjects from those that have a preference for majority voting per se (for example because they equate that with democracy and have a preference for democracy). A relevant question is whether a participant with preferences for majority voting in this sense would also consider rules 2 and 4 a reasonable compromise between selfishness and this concern for majority voting. In this case, such a participant would be indistinguishable from one who is efficiency concerned. It might be more plausible that a subject with preferences for majority voting would find rules 2 and 4 poor compromises and thus only choose between rules 1 ,

\footnotetext{
${ }^{13}$ If there was a systematic pattern that is informative about the voting strategies even if participants are not informed about the link between a valuation and a vote, e.g., if substantially more than half of the votes over time was in favor of A, then they could draw inferences about the strategies from such patterns. Given that deviations from selfish voting is small in our data, such patterns are not present.
} 
3 and 5 , depending on $\theta_{i}$ and the intensity of her preferences for majority voting. This implies that at least subjects who choose all five rules in line with efficiency concerns cannot be explained by concerns for majority voting.

Furthermore, we note that selfish behavior with error where less costly errors are more likely to be made could produce patterns of behavior that look similar to that of efficiency-concerned subjects in the sense that for large absolute valuations, rule choices are more selfish than for small absolute valuations and non-selfish rule choices are most often for the majority rule or the rule in between the selfish and the majority rule. However, efficiency concerns, but not selfishness with error would be consistent with the majority rule being chosen more often among these two rules. In the asymmetric Treatment 2 discussed below, we can distinguish between efficiency-concerned behavior and both alternatives, preferences for majority voting per se and selfishness with error.

Inequality-averse Subjects We again start with a derivation for the case that players assume others vote selfishly in the second stage. We do this to simplify the analysis and also because this is what we primarily observe. We discuss deviations from this assumption below.

Players with valuations $\theta_{i} \leq 0$ choose rule 5 and vote in favor of alternative $B$. This is both maximizing their expected payoff and minimizing inequality amongst players. Thus, for negative valuations, they behave just like selfish players (but have an additional reason to prefer $B$ ). Players with valuations $\theta_{i}>0$, however, face a trade-off. Alternative A yields a higher expected monetary payoff, but at the same time on expectation yields inequality. For small positive $\theta_{i}$, an inequality-averse player will thus still prefer B over A and choose rule 5 and vote for alternative B. For larger $\theta_{i}$, however, concerns for own income dominate concerns for inequality and the player will prefer alternative $\mathrm{A}$ and thus choose rule 1 and vote for alternative A. For example, straightforward calculation shows that for $\alpha_{i}=1$ and $\beta_{i}=0.3$ in the inequality-aversion model by Fehr and Schmidt (1999), ${ }^{14} i$ prefers $\mathrm{B}$ for $\theta_{i} \leq 1$ if he has no further information about the realization of other players' types. According to a classification made by Fehr and Schmidt based on ultimatum game data, about 40 percent of subjects satisfy $\alpha_{i} \geq 1$ and $\beta_{i} \geq 0.3$. According to an estimate of individual parameters by Blanco, Engelmann, and Normann (2011), this condition is satisfied by about 30 percent in their experiment. Note that

\footnotetext{
${ }^{14}$ According to the model by Fehr and Schmidt (1999), player $i$ has the utiltiy function $U_{i}(x)=$ $x_{i}-\frac{\alpha_{i}}{n-1} \sum_{j \neq i} \max \left\{x_{j}-x_{i}, 0\right\}-\frac{\beta_{i}}{n-1} \sum_{j \neq i} \max \left\{x_{i}-x_{j}, 0\right\}$ where $x_{k}$ is the payoff to player $k$ and $n$ is the number of players. $\alpha_{i}$ and $\beta_{i}$ thus measure player $i$ 's aversion towards disadvantageous and advantageous inequality, respectively.
} 
$\alpha_{i}=1$ implies that a subject would reject offers of less than $1 / 3$ of the total pie in an ultimatum game. Even for substantially weaker inequality aversion, one would still get preferences for B for positive $\theta_{i}$, e.g., for $\alpha_{i}=0.25$ and $\beta_{i}=0$ in the Fehr-Schmidt model (implying rejecting offers of less than $1 / 5$ in an ultimatum game), $i$ prefers $B$ for $\theta_{i} \leq 0.2$.

We can again consider alternative assumptions regarding the beliefs of subjects concerning the other participants' behavior. As argued above, rational expectations are neither a very plausible assumption, nor would that assumption make much of a difference as compared to the assumption that all vote selfishly. If an inequality-averse player $i$ expects other players to be equally inequality averse, as a strong consensus effect would predict, then $i$ would expect others with positive valuation to occasionally vote for $B$. This increases $i$ 's estimate of the average valuation conditional on $i$ being pivotal, which in turn would reduce the expected advantageous inequality but increase the expected disadvantageous inequality. Depending on $i$ 's inequality aversion parameters, this may make $A$ more or less attractive and hence change $i$ 's voting behavior for $\theta_{i}>0$. More importantly, however, it does not affect $i$ 's rule choice and voting behavior for $\theta_{i} \leq 0$. Outcome $A$ then can only yield negative payoffs and inequality and is thus never preferred over $B$. The same holds also for rational expectations or indeed all other beliefs that $i$ might have. So also for alternative assumptions regarding players' beliefs, the prediction for inequality-averse players remains that they behave like selfish players for negative valuations, but may behave differently for positive valuations. To summarize, inequality-averse players would behave as selfish players for negative and large positive valuations, but would stick with rule 5 also for small positive valuations.

\subsection{Treatment 2: Asymmetric Valuation Distribution}

With our second and third treatment we move on to our main research question whether the two-step voting procedure can increase efficiency over majority voting. We thus change the distribution of valuations such that majority voting is not ex-ante efficient. We compare Treatment 2, which features a two-step voting procedure as in Treatment 1 , with Treatment 3 where no rule choice takes place and majority voting is always im-

plemented. The game we use in these two treatments is intentionally designed to give the two-step procedure a chance to provide efficiency gains compared to the simple majority rule. However, note also that the random dictator mechanism, which we chose primarily to make the design not too complex for the participants, and because this al- 
lows us to get a clear picture of participants' preferences over rules because the random dictator mechanism is transparently incentive compatible is a rather inefficient way of aggregating preferences. Therefore, choosing a two-step procedure that more efficiently aggregates the preferences of participants regarding the voting rule may be more likely to produce efficiency gains.

\subsubsection{The Game}

In the second treatment, we have replaced the symmetric probability distribution from Treatment 1 by an asymmetric one with positive expected payoffs (of 2 Euro per player). The distribution of types is

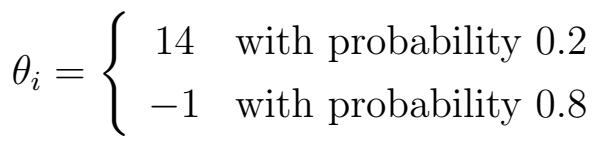

Apart from this change in the distribution of valuations, the design is identical to Treatment 1. This game has the same type of Bayesian Nash equilibrium for selfish players as the game that underlies Treatment 1 . The efficient rule here is rule 1 . Indeed the outcome that maximizes the sum of payoffs will always be reached if rule 1 is implemented and all players then vote according to their material preferences. ${ }^{15}$ Expected payoffs given rule 1 and voting according to material preferences are

$$
\begin{gathered}
0.2^{5} \cdot 14+5 \cdot 0.8^{1} \cdot 0.2^{4} \cdot \frac{4 \cdot 14-1}{5}+10 \cdot 0.8^{2} \cdot 0.2^{3} \cdot \frac{3 \cdot 14-2}{5}+10 \cdot 0.8^{3} \cdot 0.2^{2} \cdot \frac{2 \cdot 14-3}{5} \\
+5 \cdot 0.8^{4} \cdot 0.2^{1} \cdot \frac{14-4}{5}=2.327 .
\end{gathered}
$$

We note that the distribution of valuations differs is multiple respects between Treatment 1 on the one hand and Treatments 2 and 3 on the other hand. We do not, however, intend to compare behavior in Treatments 1 and 2. Rather, Treatment 1 intentionally has a fine, but symmetric distribution of valuations in order to get a first impression of the possible role of efficiency concerns, whereas Treatment 2 was designed, based on the results from Treatment 1 to have a simple distribution that makes potential efficiency gains evident, which can then be assessed by comparing Treatments 2 and 3 .

\footnotetext{
${ }^{15}$ This is a difference to Treatment 1 where the sum of payoffs is not necessarily maximized even if the ex-ante efficient simple majority rule has been chosen because a majority with small absolute valuations could outvote a minority with large absolute valuations.
} 


\subsubsection{Theoretical Predictions}

A purely selfish subject should always vote in favor of his preferred alternative in stage 2 . Moreover, he should propose rule 1 if $\theta_{i}=14$ and rule 5 if $\theta_{i}=-1$.

A sufficiently efficiency-concerned subject (i.e., one who puts at least a third of the weight on the average payoffs of the others as on her own) would choose for $\theta_{i}=-1$ rule 1 and vote for $B$. Note that efficiency concerns here impact on the rule choice, not on the voting behavior if indeed the efficient rule 1 is chosen. However, if the rule is rule 2, 3,4 , or 5 , an efficiency-concerned player with $\theta_{i}=-1$ would in equilibrium vote with positive probability for $A$. An efficiency-concerned player with $\theta_{i}=14$ would choose rule 1 and vote for $A$ unless we make the extreme assumption that he actually cares substantially more for each of the other players' earnings than his own, in which case he would choose rule 2 and vote for $A$.

An inequality-averse player $i$ acts like a selfish subject if $\theta_{i}=-1$ and also if $\theta_{i}=14$ unless $i$ 's aversion to advantageous inequality is extreme (that is for $\beta_{i}<\frac{14}{15}$ in the Fehr-Schmidt model, $i$ would choose rule 1 if $\theta_{i}=14$ and $i$ believes others vote in line with payoff maximization in second stage).

Considering alternative beliefs about others' voting behavior is not of much interest. Inequality-averse players should always vote in line with their monetary preferences (always for $\theta_{i}=-1$ and for $\theta_{i}=14$ for any rule and any beliefs about the others' behavior as long as they are not excessively inequality averse, $\beta_{i}<\frac{14}{15}$ ), as should efficiency-concerned players whenever rule 1 is chosen. So if a player assumes that others are inequality averse or that others are efficiency concerned and rule 1 is chosen, he would still expect the others to vote selfishly. If efficiency-concerned players expect others to be efficiency concerned and thus vote for $A$ with positive probability if they have $\theta=-1$ and rule $2,3,4$, or 5 is chosen, this does still not make choosing these rules preferable to choosing rule 1 and voting for $B$ (assuming all others to do so when they have $\theta=-1$ and rule 1 is chosen). The reason is that only choosing rule 1 and then voting for $B$ ensures that $A$ is chosen if and only if at least one player has $\theta=14$.

\subsection{Treatment 3: Asymmetric Valuation Distribution with Pre-determined Majority Voting}

The purpose of our third treatment is to serve as a benchmark for Treatment 2 to assess whether the two-step procedure affects welfare compared to simple majority voting, the commonly used unbiased alternative for decision making in the given context. 


\subsubsection{The Game}

Treatment 3 works with the same probability distribution as Treatment 2. However, it starts directly with the voting stage and the rule is always the simple majority rule. Hence players are informed about their valuations and then vote directly.

\subsubsection{Theoretical Predictions}

Purely selfish subjects should vote for the alternative that maximizes their payoff. Expected equilibrium payoffs are then given by

$$
0.2^{5} \cdot 14+5 \cdot 0.8^{1} \cdot 0.2^{4} \cdot \frac{4 \cdot 14-1}{5}+10 \cdot 0.8^{2} \cdot 0.2^{3} \cdot \frac{3 \cdot 14-2}{5}=0.484
$$

Efficiency-concerned subjects with a positive valuation should always vote for $A$. If all agents are sufficiently efficiency concerned, there is no sincere voting equilibrium. Instead, in a symmetric equilibrium voters with a negative valuation either play a mixed strategy (if their efficiency concerns are such that they would pay at most 1 to increase all other players' payoffs by slightly less than 2) or (if their efficiency concerns are stronger) always vote for $A$ independent of their valuation. ${ }^{16}$ Inequality-averse subjects with a negative valuation vote for alternative $B$, and with a positive valuation they vote for $A$ (if they are pivotal, then two players have $\theta=-1$ so the advantageous inequality term in the Fehr-Schmidt model is $\frac{1}{4}(15+15)=\frac{15}{2}$, so for $\beta<1$, they would not be willing to give up 14 in order to eliminate this inequality.) Hence when all agents are either selfish or inequality averse, there is an equilibrium in which all agents vote sincerely.

To summarize, sincere voting can be motivated by selfishness and inequality aversion. Insincere voting of subjects with a negative valuation is compatible with efficiency concerns but not with selfishness or inequality aversion. If subjects are sufficiently efficiency

\footnotetext{
${ }^{16}$ There is an equilibrium where altruistic players always vote for $A$ if all players are altruistic, even though this also implies that $A$ is chosen for sure when all have $\theta=-1$. If all others always vote for $A$, one is actually never pivotal and hence indifferent. Moreover, for sufficiently altruistic players, this equilibrium is trembling-hand perfect and there is also no equilibrium in mixed strategies. Assume that other altruistic players vote for $A$ with a probability smaller than 1 . Then considering the cases when one is pivotal, the expected gains in total payoffs from cases where one or two other players have $\theta=14$ exceed the expected losses when all have $\theta=-1$, so that a sufficiently efficiency concerned player would then want to vote for $A$ for sure. Hence for sufficiently strongly efficiency concerned players, voting for $A$ is weakly dominant and thus it is the only symmetric equilibrium.
} 
concerned to always vote for $A$, then the expected payoff is ${ }^{17}$

$$
\begin{aligned}
0.2^{5} \cdot 14+5 \cdot 0.8^{1} \cdot 0.2^{4} & \frac{4 \cdot 14-1}{5}+10 \cdot 0.8^{2} \cdot 0.2^{3} \cdot \frac{3 \cdot 14-2}{5}+10 \cdot 0.8^{3} \cdot 0.2^{2} \cdot \frac{2 \cdot 14-3}{5} \\
& +5 \cdot 0.8^{4} \cdot 0.2^{1} \cdot \frac{14-4}{5}+0.8^{5} \cdot(-1)=2 .
\end{aligned}
$$

The efficiency gains compared to the equilibrium with majority voting and selfish players that can be reached by efficiency concerned players with the two-step procedure thus exceed those that can be achieved through always voting for $A$ given majority voting by $\frac{(2.327-0.484)-(2-0.484)}{2-0.484}=21.57 \%$.

\subsection{Procedures}

The computerized experiments were run at the experimental laboratory mLab at the University of Mannheim with software programmed in z-Tree (Fischbacher 2007). Subjects were recruited using ORSEE (Greiner, 2015). In each session, 15 periods were played with random matching among subjects, and between 10 and 20 subjects took part. We ran three sessions each for Treatments 1, 2, and 3 for a total of 45 subjects in Treatment 1 and 55 in both Treatment 2 and 3. To avoid any income effects we did not pay all periods, but at the end of each session, one period was chosen randomly, and that period determined the payoffs of all players. If in that period alternative $A$ was chosen in subject $i$ 's group, then $i$ 's payoff was given by $\pi_{i}=\theta_{i}$, otherwise $\pi_{i}=0$. Each subject $i$ earned a show-up fee of 9 Euro plus the experimental payoff $\pi_{i}$, hence earnings could be between 2 and 16 Euro in Treatment 1 and either 8, 9 or 23 Euro in Treatments 2 and 3. In all treatments, at the end of each period, the participants learned the number of votes in favor of alternative A, the resulting outcome of the vote as well as all five valuations from their group without being able to identify the other players.

While we did not announce it in the instructions, our initial intention was to select each of the five proposals from the random dictator stage with the same probability in each period. However, due to a programming error, in Treatments 1 and 2 random ranks were calculated incorrectly and our tie-breaking rule that relied on subject identification numbers always applied. As a result, in each randomly formed group the rule that had been proposed by the subject with the lowest identification number was selected. Given

\footnotetext{
${ }^{17}$ Compared to choosing the efficient rule in Treatment 2, efficiency losses occur because $A$ is also implemented when all players have $\theta=-1$.
} 
the random matching procedure, this meant that for most subjects their proposed rule would be selected some of the time, but not always. The probabilities to be selected differed across participants, though. While this imbalance is somewhat unfortunate, we note that we have not (unintentionally) deceived our subjects because the instructions made no reference to probabilities to be selected other than that the rule would be chosen randomly.

Potentially, some subjects may have noticed that they were more or less frequently the dictator than expected given equal probabilities. Such perceived asymmetries might in principle have an impact on behavior in later periods of the experiments. However, the resulting imbalance is very unlikely to have actually played a substantial role in the decision making because subjects neither knew their identification number nor the matching scheme and hence could not predict when their rule would be chosen or note. Nevertheless, we carefully tested for any potential impact of our asymmetric matching procedure on subjects' behavior in Treatments 1 and 2. Our tests always rejected the hypothesis of a bias in Treatment 1 and with one (minor) exception also in Treatment 2. Treatment 3 was not affected by the programming error because there was no rule choice stage. A detailed discussion of these test can be found in Section 3.2.

\section{Results}

\subsection{Treatment 1}

\subsubsection{Second-stage Voting Behavior}

Voting in the second stage is overwhelmingly in line with the maximization of the subjects' own payoff. Overall, only 4.3 percent of the votes cast are in favor of the alternative which does not maximize the subject's monetary payoff (i.e., are either for A when $\theta_{i}<0$ or for $\mathrm{B}$ when $\left.\theta_{i}>0\right)$. Table 1 shows the share of deviations for different valuations. We aggregate large negative, small negative, small positive and large positive valuations, respectively, and drop $\theta_{i}=0$ because either vote is in line with payoff maximization in this case. Deviations from payoff maximization occur most frequently for small positive valuations $0.1 \leq \theta_{i} \leq 0.5$. This is where inequality aversion would predict votes against own payoff maximization, suggesting that inequality aversion may play a role for some subjects. The rate is, however, still very small with 10.4 percent. Furthermore, voting for A when $\theta_{i}<0$ is difficult to reconcile with any preference model, so it seems to result 


\begin{tabular}{cccc}
\hline \hline$-7 \leq \theta_{i} \leq-1$ & $-0.5 \leq \theta_{i} \leq-0.1$ & $0.1 \leq \theta_{i} \leq 0.5$ & $1 \leq \theta_{i} \leq 7$ \\
\hline $3.5 \%$ & $3.0 \%$ & $10.4 \%$ & $1.8 \%$ \\
\hline
\end{tabular}

Table 1: Deviations from selfish voting in the second stage

from errors. Thus taking the 3.3 percent of votes against own payoff maximization for negative valuations as an estimate of the error rate, only about 7 percent of the observations support inequality aversion, where this is expected to matter most. Remember that only a very small degrees of inequality aversion is needed to imply a preference for B when $0.1 \leq \theta_{i} \leq 0.5$.

An important question is whether participants show evidence of strategic voting, i.e., taking into account what being pivotal implies about the others' valuations. For example, if the rule implemented is rule 4 and one has a valuation of 0.1 , then one is much more likely to have a positive effect on efficiency by voting for $A$ than if rule 2 has been chosen. ${ }^{18}$ In this sense, we observed very little evidence of strategic voting. Votes are against own monetary preferences least often for rule 5 (2 percent) and most often for rule 4 (8 percent, which amounts to only 4 observations). Specifically, the rule that is in place does not have much impact on the pattern presented in Table 1, with the interesting exception that of the 16 votes most indicative of inequality aversion (for $B$ if $\left.0.1 \leq \theta_{i} \leq 0.5\right), 11$ occur when rule 1 is implemented and thus when only one vote is needed to implement $A$. In this case a player is actually pivotal only if all others vote for $B$ and are thus likely to have negative valuations. Hence $B$ is also on expectation maximizing efficiency.

\subsubsection{First-stage Behavior}

In contrast to the voting behavior in the second stage, subjects' proposals of rules in the first stage for $\theta_{i} \neq 0$ frequently deviate from the payoff-maximizing rule. Overall, 25.4 percent of the proposals made do not coincide with a payoff-maximizing voting rule. According to Table 2, which shows the rate of deviations from the selfish rule and again groups valuations into large negative, small negative, small positive and large positive valuations, there is a clear pattern. First, deviations from the payoff-maximizing rule are more frequent for small absolute valuations than for large absolute valuations, in line

\footnotetext{
${ }^{18}$ Exact predictions how voting behavior should depend on the implemented rule require assumptions regarding participants' beliefs about other participants' voting behavior. Nevertheless, except for very specific beliefs, strategic voting should lead to differences in voting behavior for different rules.
} 


\begin{tabular}{cccc}
\hline \hline$-7 \leq \theta_{i} \leq-1$ & $-0.5 \leq \theta_{i} \leq-0.1$ & $0.1 \leq \theta_{i} \leq 0.5$ & $1 \leq \theta_{i} \leq 7$ \\
\hline $14.6 \%$ & $39.3 \%$ & $32.5 \%$ & $18.8 \%$ \\
\hline \hline
\end{tabular}

Table 2: Deviations from selfish voting in the first stage

with any model that combines selfishness with some other motive. Second, deviations are about equally likely for negative than for positive valuations, a pattern that is in line with efficiency concerns (or selfishness with error or preferences for majority voting). ${ }^{19}$

A key prediction of our efficiency-concerns model and key distinction to the predictions by inequality aversion are that deviations from the selfish rule are not only decreasing in absolute valuation but are also symmetric around 0 . In order to assess this prediction, we show the distribution of the chosen rules for each $\theta_{i}$ in Table 3 . Looking at the disaggregated distribution, we see that for $\left|\theta_{i}\right| \geq 1$, the vast majority of choices (79.7 percent or more) are for the selfish rule, while the remaining choices are spread fairly evenly across the other rules, which would be in line with noise. In contrast, for $\left|\theta_{i}\right|<1$, while still more than half of the rule choices are for the selfish rule, a substantial share is in each case in favor of the efficient rule 3 or the rule in between the selfish and the efficient rule (between 20.4 percent and 36.2 percent, overall 28.7 percent). As we can see, the deviations from the selfish rule are quite symmetric. This pattern is in line with efficiency concerns. To test for the symmetry of the distribution, we compare for each valuation $\theta_{i}$ the distribution of chosen rules with the inverted distribution for $-\theta_{i}$. According to Fisher exact tests, the distributions are not significantly different for any $\theta_{i} \in\{0.1, \ldots, 7\}(p>10 \%$ in each case $){ }^{20}$

${ }^{19}$ One obvious reason why there are more deviations from selfishness in the first stage than in the second stage may be that finding the selfish rule is more complex. Furthermore, for the choice of rules, there is only one way to be "right" (i.e., payoff maximizing) but four ways to be "wrong". Both these arguments would suggest that pure confusion may contribute to the higher share of deviations from selfishness in the first stage. However, the smaller rate of deviations for larger absolute valuations suggests that the non-selfish rules are not chosen plainly out of confusion. While errors being rarer for larger absolute valuations is consistent with standard models of choice with error, the pattern of rules that are chosen for small absolute nonzero valuations suggests that the deviations from the selfish rule are not only the result of confusion. Specifically, some rules make sense given our model of efficiency concerns, namely those that range from the selfish rule to majority voting whereas others do not, namely those that are biased against the maximization of subjects' own payoff, i.e., the chosen rule favors alternative A (rules 1 and 2) while the subject has a negative valuation or it favors B (rules 4 and 5) while the subject has a positive valuation. We find only about 7 percent of the latter type of rule choices in each of the four groups of valuations.

${ }^{20}$ Note that the rules are on an ordinal scale. The averages in Table 3 serve primarily for illustration. Note also that the Fisher test does not correct for possible dependence of observations due to repeated observations for the same person and within the same group. Given that we demonstrate here the absence of a significant difference, this is arguably not problematic. 


\begin{tabular}{cccccccc}
\hline \hline$\theta_{i}$ & $\sum$ & 1 & 2 & 3 & 4 & 5 & average rule \\
\hline-7 & 59 & 4 & 0 & 4 & 1 & 50 & 4.57 \\
-3 & 51 & 2 & 2 & 3 & 0 & 44 & 4.61 \\
-1 & 61 & 1 & 3 & 3 & 2 & 52 & 4.66 \\
-0.5 & 42 & 1 & 2 & 3 & 8 & 28 & 4.43 \\
-0.2 & 46 & 1 & 3 & 5 & 11 & 26 & 4.26 \\
-0.1 & 47 & 2 & 0 & 11 & 6 & 28 & 4.23 \\
0 & 45 & 7 & 1 & 22 & 2 & 13 & 3.29 \\
0.1 & 49 & 32 & 3 & 7 & 3 & 4 & 1.86 \\
0.2 & 46 & 29 & 9 & 4 & 0 & 4 & 1.72 \\
0.5 & 59 & 43 & 5 & 10 & 0 & 1 & 1.49 \\
1 & 59 & 47 & 2 & 5 & 3 & 2 & 1.49 \\
3 & 57 & 46 & 3 & 7 & 1 & 0 & 1.35 \\
7 & 54 & 45 & 1 & 3 & 4 & 1 & 1.43 \\
\hline \hline
\end{tabular}

Table 3: Distribution and average of proposed rules for the different valuations in Treatment 1.

Rule choices that are biased against own monetary preferences are not chosen more often for $0<\theta_{i} \leq 0.5$, where they would be in line with inequality aversion than for $\theta_{i} \geq 1$ or $\theta_{i}<0$. We also see that for $\theta_{i}=0$ the most frequently chosen rule is 3 , as predicted by efficiency concerns, and not 5 , which would be predicted by inequality aversion. Hence, Table 3 provides very little evidence in favor of inequality aversion playing a role for subjects' rule choices. We also note that the median rule is always the selfish rule, so even for very small own monetary gains or losses, an absolute majority always goes for the selfish rule. The efficiency concerns model is also supported by ordered probit regressions of the rank number of the chosen rule and probit regressions for a dummy whether subjects are choosing a non-selfish rule (see Appendix C)

Deviations from selfish behavior do not decrease substantially over time (see Figure 1). Except for a drop of non-selfish rule choices from the first to the second period, there is no clear discernible time trend. Hence, deviations from the selfish prediction are unlikely exclusively errors or driven by expectations of reciprocal behavior, because both would imply a decreasing time trend. ${ }^{21}$

For the symmetric distribution of valuations, the simple majority rule is efficient and given an exogenously chosen majority rule, selfish voting is efficient. Thus, because the

\footnotetext{
${ }^{21}$ If we look at the sessions of Treatment 1 separately, the pattern is very similar in all three of them. The fluctuations are more extreme, however, due to the smaller number of observations. Most importantly, neither of the sessions shows a clear upward or downward trend in either of the three measures.
} 


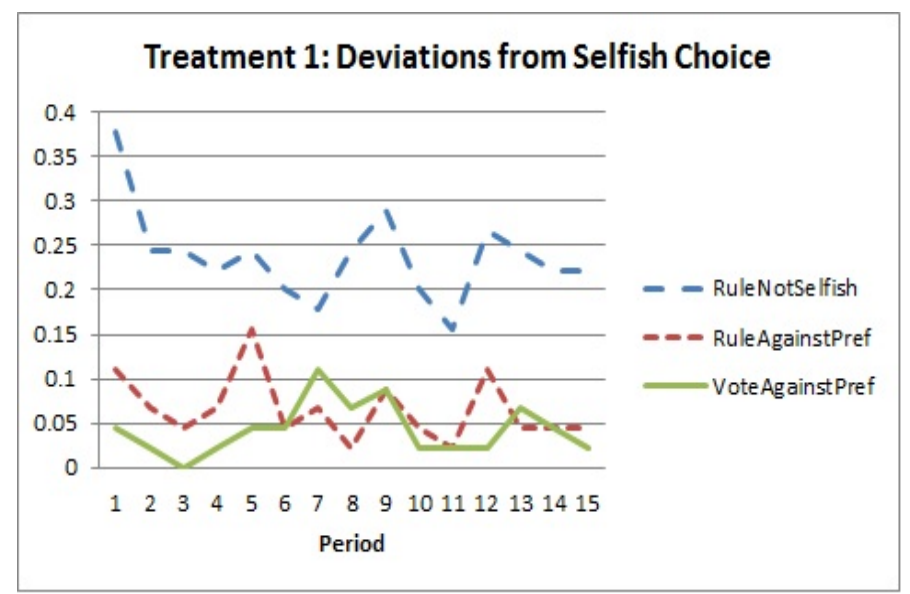

Figure 1: Share of choices that differ from the selfish prediction across periods. VoteAgainstPref corresponds to votes for $A$ given $\theta<0$ or votes for $B$ given $\theta>0$. RuleNotSelfish summarizes all rule choices that are not for rule 5 when $\theta<0$ or not for rule 1 when $\theta>0$. RuleAgainstPref is the subset where the rule is biased against the subject's own monetary preferences, i.e., for rule 1 or 2 when $\theta<0$ or for rule 4 or 5 when $\theta>0$.

random dictator rule that we employ cannot result in a rule that is ex-ante more efficient than the majority rule, ${ }^{22}$ but often results in less efficient rules, it is not surprising that the total payoffs in Treatment 1 are about 35 percent lower than what would be obtained with pre-determined majority voting and selfish voting behavior given the draws of valuations we observe. However, given the rules of our experiment, the deviation from selfish behavior leads to total payoffs being about 8 percent higher than the payoff that selfish behavior on both stages would yield given the draws of valuations realized in the experiment. We further investigate this second, positive welfare effect in Treatments 2 and 3.

\subsection{Treatments 2 and 3}

We again start by considering voting behavior in the second stage. In the asymmetric Treatment 2 with rule-choice stage, the observed voting behavior of subjects with positive valuations $\left(\theta_{i}=14\right)$ is consistent with selfishness as well as with efficiency concerns (as well as non-extreme forms of inequality aversion). Only in 3 percent of cases, subjects

${ }^{22}$ Though at an interim-stage if the valuation of the dictator is $\theta=7$ or $\theta=-7$, it might result in the more efficient rules 2 and 4 , respectively. 
with $\theta_{i}=14$ voted for alternative $B$. This would correspond to the same error rate as observed in Treatment 1 . Subjects with $\theta_{i}=-1$ vote for alternative $A$ in 13.6 percent of the cases. Their behavior is inconsistent with selfishness and inequality aversion, but is consistent with efficiency concerns if the rule is not rule 1 . Note, again, that the rate of non-selfish votes is relatively low, given that quite some efficiency gains are possible, so even moderately efficiency-concerned players could be expected to vote for $A$ if the rule is not rule 1 .

Interestingly, subjects' voting behavior is statistically independent of the implemented voting rule. ${ }^{23}$ This is inconsistent with efficiency concerns because these imply for $\theta_{i}$ $=-1$ to vote for $A$ only for rules unequal to rule 1 . Not very surprisingly, though, voting behavior is correlated with subjects' own rule choice. In 97 percent of the cases where subjects choose the selfish rule, they vote for the outcome that maximizes their own payoff (so they are consistently selfish), but in between 19 and 33 percent of the cases where subjects choose a non-selfish rule do they vote against their monetary preference in the second stage.

Regarding the rule-choice stage, subjects with a valuation of $\theta_{i}=14$ choose the selfish rule (rule 1) in 88 percent of cases (see Table 4). Among the 12 percent remaining proposals, 6 percent are for the simple majority rule. The latter observation indicates that these subjects may have a preference for democracy - interpreted as a preference for majority voting. Subjects with a valuation of $\theta_{i}=-1$ choose the selfish rule (rule 5) in only 56 percent of cases and the efficient rule (rule 1) in 19 percent of cases. The behavior of these 19 percent is inconsistent with selfishness and inequality aversion, but in line with efficiency concerns. Furthermore, 9 percent choose the simple majority rule, which could suggest a preference for majority voting per se or for mild efficiency concerns. While we thus observe that substantially more subjects deviate from the selfish rule if they have $\theta_{i}=-1$ than if they have $\theta_{i}=14$, still a majority chooses the selfish rule 5 and in 97 percent of the cases then also votes for $B$ and thus enforces $B$ even if all other subjects have $\theta_{i}=14$, which amounts to quite substantial selfishness.

\footnotetext{
${ }^{23}$ Specifically, for rules $1,2,3$, and 5 , the share of votes for $A$ by subjects with $\theta_{i}=-1$ is between 12.6 percent and 14.8 percent. Only for rule 4 the share is lower with just 4.7 percent. However, there are only 21 observations for subjects with $\theta_{i}=-1$ when rule 4 is implemented. According to Fisher's exact test, the distribution of votes does not differ between rule 4 and any of the other rules, or between rule 4 and all other rules pooled or between any pair of rules $(p>0.3$ for all two-sided tests). Note that this test does not correct for dependence due to multiple choices of the same individual and interaction within sessions. Neglecting the dependence would rather lead to identifying significance where there is none, so since the point here is to show that there is no dependence of voting on the rule, this does not appear problematic.
} 


\begin{tabular}{cccccc}
\hline \hline$\theta_{i}$ & 1 & 2 & 3 & 4 & 5 \\
\hline-1 & 123 & 72 & 58 & 38 & 365 \\
14 & 149 & 5 & 10 & 4 & 1 \\
\hline \hline
\end{tabular}

Table 4: Distribution of proposed rules for both valuations in Treatment 2.

Regarding possible impacts of the imbalanced probability of one's proposed rule being chosen in Treatments 1 and 2, we test with logistic regressions (clustering standard errors on the subject level and controlling for the valuation) whether a certain rule is more likely to be chosen by participants who are likely to be the rule dictator than by those who are less likely to be the rule dictator. We employ four different measures of the relative likelihood to be the rule dictator. First, the subject ID, as the probability of being chosen as rule dictator is decreasing in the ID. Second, a dummy for a subject ID $\leq 5$, which captures those being relatively likely to be chosen as rule dictator. Third, a dummy whether the subject is the rule dictator in the current period, which captures the likelihood of being chosen in the aggregate across periods by giving more weight to those subjects who are more frequently chosen as the rule dictator. Fourth, the relative frequency with which a subject has been the rule dictator in the previous periods. The latter measure would capture if subjects had noticed that they had been chosen as rule dictator surprisingly often or surprisingly rarely and this had affected their behavior. The only significant difference (at $p<0.05$ ) that we find is that in Treatment 2 , having more frequently been the rule dictator in past periods decreases the likelihood of choosing majority voting. Given that we run a total of 40 tests (2 treatments $* 4$ measures $* 5$ rules), one significant difference is to be expected by chance and not likely an indication that our unbalanced design affected rule choices. Moreover, the one significant difference affects the choice of neither the selfish nor the efficient rule, which would be more reason to worry. $^{24}$

The actual average payoff in Treatment 2 per period was $W_{a}=1.30$. This value

\footnotetext{
${ }^{24}$ It is not too surprising that the likelihood to be the rule dictator did not have an impact on rule choices. First, inferring the actual asymmetry during the experiment was very difficult because subjects never learned whether their personal choice was actually implemented. Even for those for whom this was never the case, the implemented rule often coincided with their choice. Furthermore, with equal probabilities to be selected, during the first periods, the probability not to be selected is substantial, declining from 80 percent in period 1 to 33 percent in period 5. Hence it would take a sizable number of periods to realize that one's own choice is chosen with lower frequency. Three participants in Treatment 1 noted in a post-experimental questionnaire that they were surprised that their choice was (almost) always implemented, but none remarked that their rule choice was surprisingly rarely implemented (which would be more of a concern because this would undermine incentives). No such remarks were made in the post-experimental questionnaire in Treatment 2.
} 
exceeds the hypothetical average payoff with majority rule, selfish voting behavior, and the observed valuation distribution, which is $W_{s}=0.44$. Thus the combination of letting subjects choose the rule and their deviation from selfishness yields a substantial efficiency gain. Part of this gain stems from the fact that the efficient rule is sometimes chosen, part of it results from non-selfish voting when the rule is not rule 1 . We thus also compare the average payoff to the hypothetical average payoff that would result under exogenously chosen majority rule given the observed valuation draws and the average voting behavior observed if the majority rule was chosen in the experiment, which is $W_{o}=0.90$. Since $W_{o}>W_{s}$, but $W_{o}<W_{a}$, the observed efficiency gains are derived indeed both from the choice of the efficient rule and from non-selfish voting. We also note that the efficient rule is sometimes chosen because a player with $\theta_{i}=14$ is chosen as the dictator. Hence the choice of the efficient rule is not exclusively based on altruism, but sometimes also simply on allowing somebody with strong interests to have his will.

The comparison of Treatments 2 and 3 allows us to assess whether our two-stage decision process leads to efficiency gains compared to actual behavior given an exogenously given majority rule. Using an OLS regression with robust standard errors (controlling for the number of positive valuations in the group), we find that with rule-choice stage, the average individual payoff is about 0.47 higher per period than under exogenously given majority voting. This difference is far from significant $(p>0.5)$. It is also smaller than what one would expect from looking at the behavior in Treatment 2. The reason is that for exogenously given majority rule, voting behavior is less selfish than observed with the two-stage procedure. Specifically, the probability to vote for $\mathrm{A}$ for $\theta_{i}=-1$ is by 11 percentage points lower in Treatment 2 with rule choice than in Treatment 3. According to a linear probability model, this difference is highly statistically significant $(p<0.01$, also if standard errors are clustered at group level). Hence in Treatment 3 with pre-determined majority voting, efficiency gains are rather obtained by non-selfish voting, but in Treatment 2 with rule-choice stage by choosing the efficient rule. This suggests that subjects consider non-selfish behavior at the two stages partly as substitutes. They may engage in moral licensing, voting selfishly in the second stage if they have chosen a non-selfish rule, even if that rule was not chosen.

\subsection{Interpretation of the Results from Treatments 1-3}

We observe a non-trivial share of non-selfish choices on the rule-choice stage of Treatment 1 that is consistent with efficiency concerns. Similarly, in the asymmetric Treat- 
ment 2, 19 percent of the subjects' rule proposals given a negative valuation can be explained by efficiency concerns, and another 25 percent would be consistent with at least some degree of efficiency concerns. In the second stage, however, in only 14 percent of cases, subjects with negative valuation vote for alternative $A$ in Treatment 2 . This share is higher in Treatment 3, with exogenously given majority rule. Since somewhat, but not very strongly, efficiency-concerned subjects should actually consider mixed strategies in Treatment 3, this may constitute a lower bound on the share of efficiency-concerned individuals in Treatment 3.

Interestingly, in both Treatment 1 and 2, the share of own-payoff maximizing votes in the second stage is higher than the share of own-payoff maximizing choices in the rule-choice stage. While this may reflect that efficiency concerns have little effect in the voting stage as argued above, it might also be the case that subjects approach the two stages differently and do not behave consistently with the same preference model across both stages. Partly, this may reflect moral licensing, as subjects who have chosen a nonselfish rule feel justified to cast a selfish vote, even if a different rule has been chosen. This would also be in line with the higher share of non-selfish votes in Treatment 3 than in Treatment 2 (if the majority rule is chosen in the latter) because in Treatment 3 such moral licensing does not apply as no rule is chosen. Alternatively, subjects may perceive the rule-choice stage as a more principled decision, where they should do the "right thing", whereas voting selfishly in the second stage is perhaps considered more acceptable. This would, however, not explain the higher share of non-selfish votes in Treatment 3 .

We address this apparent inconsistency in two additional treatments (Treatments 4 and 5 reported in Appendix A), where we eliminate uncertainty about what it means to be pivotal and can thus properly test for inconsistent behavior across the two stages, independent of assumptions about beliefs. While the results from these treatments should be considered with some care because several participants had substantial difficulty understanding the complex design, they do provide further indication that behavior in the voting stage is more frequently selfish than in the rule-choice stage.

From the comparison of Treatments 1 and 2 we see that subjects very clearly react to the distribution of valuations. In particular, there are more choices for the efficient rule in Treatment 2 where efficiency gains are higher. This change in behavior is in line with subjects being concerned with the maximization of total payoffs, but not with a preference for majority voting per se or with a selfishness with noise model. 


\section{Discussion and Concluding Remarks}

Our paper experimentally addresses a fundamental problem in political economics. Voting rules should adjust to the problem at hand while frequently, they are fixed in practice. Our results indicate that a non-trivial share of voters are willing to efficiently adjust the voting rule even when they are already privately informed about their preferences regarding the voting outcome. Choosing in a two-step procedure, i.e. choosing a rule first and then voting according to this rule, can yield higher aggregate payoffs than simple majority voting. Our results therefore suggest that it may be useful to let voters fix or alter voting thresholds in referenda.

Another important finding of our paper is that when choosing rules, different types of social preferences may matter than when choosing outcomes. Efficiency appears to be a more important behavioral motive when subjects decide on procedures than if they decide on outcomes according to a given procedure. This effect could in principle be exploited to increase expected payoffs in democratic decisions or to end political gridlock in reform processes.

How can we interpret that apparently subjects are more likely to deviate from selfish maximization of monetary payoffs in the rule-choice stage than in the voting stage? A first possible explanation is that at the rule-choice stage, the final outcome is still somewhat remote and this may reduce the temptation to act selfishly and hence make subjects choose somewhat more altruistically. A second possibility is that at the rulechoice stage, at least some participants believe they should choose what is the right thing to do, and choosing the efficient rule is likely perceived as the right thing to do. In spite of the fact that rules are chosen at an interim stage, choosing a rule may have a flavor of choosing what is best at an ex-ante stage, or put differently, even though rule choice does not happen behind a veil of ignorance, participants may think that it should. In the voting stage, however, voting in line with one's own monetary preferences is likely to be perceived as legitimate. Voting selfishly is quite generally accepted, whereas tilting rules in one's favor is not. Therefore, a simple outcome-oriented preference model is unable to adequately capture behavior across both stages in our two-stage voting game.

Voting mechanisms where players first decide about the majority threshold are related to the concept of liberal paternalism (Thaler and Sunstein, 2008). A social planner might know that given the distribution of valuations, the optimal voting threshold may deviate from the simple majority rule. However, he may lack the political legitimacy to implement this rule. Instead, he may thus leave the decision regarding the threshold 
to the participants, who will, if they are sufficiently efficiency concerned, choose the optimal threshold. This allows for gains compared to exogenously assigned majority voting. These gains can be increased, if the rule choice optimally exploits the efficiency concerns of those who have any.

In our asymmetric treatment we found that in about 44 percent of the cases subjects suggested a rule that was not completely biased in favor of alternative $B$. This indicates that an appropriately designed majority rule for the choice of the voting rule may produce better results than our simple random dictator mechanism that allows a randomly chosen person to implement her preferred choice. Testing and comparing such more complex multi-stage mechanisms that make use of more than just one subject's proposal is worth additional research.

\section{References}

[1] Austen-Smith, D. and J.S. Banks (1996). "Information Aggregation, Rationality, and the Condorcet Jury Theorem." American Political Science Review, 90, 34-45.

[2] Balafoutas, L., M.G. Kocher, L. Putterman, and M. Sutter (2013). "Equality, Equity and Incentives: An Experiment." European Economic Review 60, 32-51.

[3] Barbera, S. and M.O. Jackson (2004). "Choosing How to Choose: Self-Stable Majority Rules and Constitutions." Quarterly Journal of Economics 119, 1011-1048.

[4] Bergemann, D. and S. Morris (2005). "Robust Mechanism Design." Econometrica $73,1771-1813$.

[5] Bergemann, D. and S. Morris (2012). Robust Mechanism Design: The Role of Private Information and Higher Order Beliefs. World Scientific, Singapore.

[6] Bierbrauer F. and M. Hellwig (forthcoming). "Robustly Coalition-Proof Incentive Mechanisms for Public Good Provision are Voting Mechanisms and Vice Versa." Review of Economic Studies.

[7] Blanco, M., D. Engelmann, and H.-T. Normann (2011). "A Within-Subject Analysis of Other-Regarding Preferences." Games and Economics Behavior 72, 321-338.

[8] Blais, A., J.-F. Laslier, F. Poinas, and K. van der Straeten (2015). "Citizens' Preferences about Voting Rules: Self-Interest, Ideology, and Sincerity." Public Choice 164, 423-442.

[9] Bolton, G. E., and A. Ockenfels (2000). "ERC: A Theory of Equity, Reciprocity, and Competition." American Economic Review 90, 166-193. 
[10] Börgers, T. and P. Postl (2009). "Efficient Compromising." Journal of Economic Theory 144, 2057-2076.

[11] Brennan, G. and J.M. Buchanan (1985). "The Reason of Rules: Constitutional Political Economy." Cambridge: Cambridge University Press.

[12] Casella, A. (2005). "Storable Votes." Games and Economic Behavior 51, 391-419.

[13] Casella, A., A. Gelman, and T.R. Palfrey (2006). "An Experimental Study on Storable Votes." Games and Economic Behavior, 57, 123-154.

[14] Charness G. and M. Rabin (2002). "Understanding Social Preferences with Simple Tests." Quarterly Journal of Economics 117, 817-869.

[15] Cramton, P., R. Gibbons, and P. Klemperer (1987). "Dissolving a Partnership Efficiently." Econometrica 55, 615-632.

[16] Engelmann, D. and V. Grimm (2012). "Mechanisms for Efficient Voting with Private Information about Preferences." Economic Journal 122, 1010-1041.

[17] Engelmann, D. and M. Strobel (2000). "The False Consensus Effect Disappears if Representative Information and Monetary Incentives are Given." Experimental Economics 3, 241-260.

[18] Engelmann, D. and M. Strobel (2004). "Inequality Aversion, Efficiency and Maximin Preferences in Simple Distribution Experiments." American Economic Review $94,857-869$.

[19] Feddersen, T.J. and W. Pesendorfer (1998). "Convicting the Innocent: The Inferiority of Unanimous Jury Verdicts under Strategic Voting." American Political Science Review 92, 23-35.

[20] Fehr, E. and K.M. Schmidt (1999). "A Theory of Fairness, Competition, and Cooperation." Quarterly Journal of Economics 114, 817-868.

[21] Fischbacher, U. (2007). "z-Tree: Zurich Toolbox for Ready-made Economic experiments." Experimental Economics 10, 171-178.

[22] Güth, W. and M.F. Hellwig (1986). "The Private Supply of a Public Good." Journal of Economics 5, 121-159.

[23] Greiner, B. (2015). "Subject Pool Recruitment Procedures: Organizing Experiments with ORSEE." Journal of the Economic Science Association 1, 114-125.

[24] Grüner, H.P. and Y. Koriyama (2012). "Public Goods, Participation Constraints, and Democracy: A Possibility Theorem." Games and Economic Behavior 75, 152167. 
[25] Guarnaschelli, S., R.D. McKelvey, and T.R. Palfrey (2000). "An Experimental Study of Jury Decision Rules." American Political Science Review 94, 407-423.

[26] Hortala-Vallve, R. and A. Llorente-Saguer (2010). "A Simple Mechanism for Resolving Conflict." Games and Economic Behavior, 70, 375-391.

[27] Jackson, M.O. and H.F. Sonnenschein (2007). "Overcoming Incentive Constraints by Linking Decisions." Econometrica 75, 241-258.

[28] Kaplan, T.R. and B.J. Ruffle (2012). "Which Way to Cooperate." Economic Journal $122,1042-1068$.

[29] Kosfeld, M., A. Okada, and A. Riedl (2009). "Institution Formation in Public Goods Games", American Economic Review 99, 1335-1355.

[30] Ledyard, J.O. T. R. Palfrey (2002) "The Approximation of Efficient Public Good Mechanisms by Simple Voting Schemes." Journal of Public Economics 83, 153-171.

[31] Masatlioglu, Y., S. Taylor, and N. Uler (2012). "Behavioral Mechanism Design: Evidence from the Modified First-Price Auction", Review of Economic Design 6, 159-173.

[32] Myerson, R. (1981). "Optimal Auction Design." Mathematics of Operations Research $6,58-73$.

[33] Myerson, R. (1983). "Mechanism Design by an Informed Principal." Econometrica $51,1767-1797$.

[34] Myerson, R. and M. Satterthwaite (1983). "Efficient Mechanisms for Bilateral Trading." Journal of Economic Theory 29, 265-81.

[35] Oleszek, W.J. (2014). "Congressional Procedures and the Policy Process." $9^{\text {th }}$ Edition. Washington DC: CQ Press.

[36] Rawls. J (1972). "A Theory of Justice." Oxford: Clarendon Press.

[37] Ross, L., D. Greene, and P. House, (1977). "The 'False Consensus Effect': An Egocentric Bias in Social Perception and Attribution Processes." Journal of Experimental Social Psychology 13, 279-301.

[38] Schmitz, P. W. (2002). "Simple Contracts, Renegotiation under Asymmetric Information, and the Hold-up Problem." European Economic Review 46, 169-188.

[39] Schmitz, P.W. and T. Tröger (2012). "The (Sub-)Optimality of the Majority Rule." Games and Economic Behavior 74, 651-665.

[40] Segal, I. and M. Whinston (2011). "A Simple Status Quo that Ensures Participation (with Application to Efficient Bargaining)." Theoretical Economics 6, 109-125. 
[41] Thaler., R.H. and C.R. Sunstein (2008). "Nudge: Improving Decisions about Health, Wealth, and Happiness." Yale University Press.

[42] Trebbi, F., P. Aghion, and A. Alesina (2008). "Electoral Rules and Minority Representation in U.S. Cities". Quarterly Journal of Economics 123, 325-357.

[43] Weber, M. (2016). "Choosing the Rules: Preferences over Voting Systems in Assemblies of Representatives." mimeo.

[44] Wilson, R. (1985). "Incentive Efficiency in Double Auctions". Econometrica 53, 1101-1115. 


\section{Appendix}

\section{A. Testing for Inconsistent Behavior across Stages}

\section{A.1. Motivation and Experimental Design}

The results of Treatments 1 and 2 indicate that several participants behave differently across both stages. In particular, some participants vote selfishly in the second stage but propose efficient rules in the first stage of the game. This raises the question whether subjects' behavior is consistent with utility maximization across stages or whether each stage appeals to a different behavioral motive in the sense that subjects act more altruistically in the rule-choice stage than in the voting stage. Our last two treatments are designed to rigorously test for inconsistencies across stages. In particular, Treatment 4 implements the following game where we do not need to worry about beliefs of subjects about the other subjects' rule choices and hence whether the chosen rule reveals something about what being pivotal implies for the other subjects' valuations. Treatment 5 is a simplified variant that will be explained below.

In this game we consider three players. First, nature determines players' valuations, where $\theta=-1$ or $\theta=14$, both with probability $\frac{1}{2}$ (because the game is rather complicated, we decided to simplify the assignment of valuations and both outcomes being equally likely is easier to explain and understand than one occurring with probability $\left.\frac{4}{5}\right)$. Then, as in the other treatments a two-stage voting procedure follows. In the first stage a rule is suggested by each player. Each player's choice is limited to rules 1 and 2 , i.e., rules that require either one or two votes to pass A. ${ }^{25}$ Then either one player's rule choice is randomly selected to be implemented (with probability $\frac{1}{4}$ for each player's rule choice), or rule 2 is randomly chosen to be implemented as the default, which also happens with probability $\frac{1}{4}$. In the second stage of the voting procedure, all players cast their intended votes in favor of alternative $A$ or $B$. However, only one randomly chosen subject can freely decide how to vote. The others are "forced" to vote in line with their monetary preferences, i.e., to vote for $B$ if $\theta<0$ and to vote for $A$ if $\theta>0$. To gather more data, all vote, but all but one of the votes will be replaced with the money-maximizing vote.

We represent a strategy of a player who has a given valuation $\theta$ by a vector $\left(R, v_{1}, v_{2}, v_{c}\right)$, where $R \in\{1,2\}$ denotes the rule proposed, and $v_{1}, v_{2}, v_{c} \in\{A, B\}$ denote the vote un-

\footnotetext{
${ }^{25} \mathrm{~A}$ similar argument could be made by restricting the rule set to rules 2 and 3.
} 
der rule 1 , rule 2 proposed by a player, or rule 2 selected by the computer, respectively. Our test for consistency is based on the assumptions that (i) all subjects expect players with a positive valuation to always choose rule 1 and to vote in favor of alternative $A$ for any implemented rule and (ii) all players with a negative valuation vote in favor of alternative $B$ if rule 1 is implemented, and all players expect the others to do so.

We first show that a player $i$ who proposes rule 1 even though $\theta_{i}<0$ weakly prefers alternative $A$ in case that exactly one other player has a positive valuation. A player $i$ with $\theta_{i}<0$ who proposes rule 1 chooses, under the above assumption (ii), strategy $(1, B, B, B)$, or $(1, B, A, A)$, or $(1, B, B, A)$, or $(1, B, A, B)$ (under the standard assumption that by the time they propose a rule the players actually have a complete plan how to vote contingent on all possible implemented rules, an assumption that may well be violated for experimental participants).

Consider first strategy $(1, B, B, B)$. Alternative $A$ is chosen if (i) rule 1 is selected and exactly one other subject has a valuation $\theta>0$ or (ii) two other subjects have a valuation $\theta>0$ or (iii) exactly one other subject has $\theta>0$ and one other subject with $\theta<0$ votes for $A$ and her vote is chosen to count. Compare this to the strategy $(2, B, B, B)$. Here, alternative $A$ is chosen under the same conditions as above. Since rule 1 obtains with a lower probability under the second strategy and if exactly one other player has $\theta>0, A$ is more likely to result under rule 1 than under rule $2,{ }^{26} i$ must be weakly in favor of alternative $A$, conditional on exactly one player having a positive valuation, if $i$ chooses $(1, B, B, B)$ rather than $(2, B, B, B)$. The same reasoning holds for comparing strategy $(1, B, B, A)$ to $(2, B, B, A)$ because this only possibly leads to a different outcome if rule 2 was chosen by default, which is beyond the control of the players. For strategies $(1, B, A, A)$ and $(1, B, A, B)$ the same argument applies unless player $i$ believes that both other players vote for $A$ for sure if rule 2 is chosen by a player. For such a belief, the outcome would actually always be the same for $\left(1, B, A, v_{c}\right)$ and $\left(2, B, A, v_{c}\right)$ because in that case both when rule 1 is implemented and when rule 2 , chosen by a player, is implemented, $A$ will result whenever at least for one player $\theta>0$. Thus we make the mild additional assumption that $i$ does not assume that with certainty both other players are sufficiently altruistic such that they will vote for $A$ even if they have $\theta<0$ when rule 2 is implemented.

Now consider $i$ in the voting stage when the rule has been determined to be rule

\footnotetext{
${ }^{26}$ Note that given that $i$ votes for $B$ under rule 2, and her own vote may be chosen to count, $A$ will not result for sure if exactly one other player has $\theta>0$, even if $i$ assumes the remaining player would vote for $A$ under rule 2 for sure.
} 
2 , not chosen by any subject, so $i$ has not learned anything about the other subjects' valuations. If $i$ 's vote is relevant, then the other two players are forced to vote according to their monetary preferences. Thus $i$ is pivotal and voting for $A$ implements $A$ if exactly one of the other players has $\theta>0$. Thus if $i$ prefers $A$ when exactly one of the others has $\theta>0$, then $i$ should vote for $A$. Hence if $i$ chooses rule 1 , but votes for $B$ if the rule is exogenously chosen to be rule 2 , then this is inconsistent with our model, but more generally with any model that is consistent with choosing rule 1 in the first place (which must include some form of altruism).

Note that even if player $i$ thinks the others are altruists who would want to vote for $A$ given rule 2 to help one player with a positive payoff, $i$ does not have an incentive to strategically choose rule 1 for $\theta_{i}<0$. Given that only one player can freely choose in the voting stage, it cannot happen given rule 2 that $A$ gets implemented even though both other players have a negative valuation but are altruistic. Thus given rule $2, A$ can only result if at least one of the others has a positive valuation. But if that is the case, $A$ also results if $i$ chooses rule 1 (unless the player with a positive valuation is chosen to be the one who can vote and votes for $B$, which is unlikely), and $A$ will also result if rule 1 is chosen and a confused altruist's vote is chosen to count. Thus if $\theta_{i}<0$ and $i$ wants $B$, then $i$ should choose rule 2 .

Because the participants exhibited substantial problems in understanding the instructions in Treatment 4, which implemented this game, Treatment 5 was based on a simplified variant. Specifically, the option that the computer automatically implements rule 2 with probability $\frac{1}{4}$ was eliminated. This simplifies the description of the first stage substantially. In the first stage now simply all three players choose a rule and one of them is then randomly selected and her chosen rule is implemented. The second stage remains unchanged, because for the test of inconsistency, it is essential that players know that if their vote counts, the other two players are voting in line with their monetary preferences. Simplifying the first stage now means that if a player learns that rule 2 has been implemented, he knows that this has been chosen by another player. Since it is highly implausible that a player with $\theta=14$ chooses rule 2 , the player should thus infer that another player has $\theta=-1$. Nevertheless, if rule 2 is implemented, a player can still infer that he is only pivotal (in the sense that his vote is chosen to count and changes the outcome) if exactly one other player has $\theta=14$. Hence if he prefers outcome $A$ in that case, he should vote for $A$. Regarding the choice in the first stage, the same argument as above holds, that is a player with $\theta=-1$ should choose rule 1 only if he prefers $A$ if exactly one player has $\theta=14$. Hence a player with $\theta=-1$ choosing rule 1 but voting 
for $B$ if rule 2 is implemented behaves inconsistently in the sense that his first-stage rule choice suggests a higher degree of altruism than his second-stage vote. The only difference between Treatments 4 and 5 is that in the latter, a player might (implausibly) assume that a player with $\theta=14$ will choose rule 2 and hence conclude from the implementation of rule 2 that at least one other player has $\theta=14$. However, this does not change anything about the conclusions from being pivotal. A wrong conditioning on being pivotal might possibly make such a player be more inclined to vote for $A$ if rule 2 is implemented (because he now "knows" that one other player has $\theta=14$ rather than inferring that from being pivotal). This, however, only strengthens the argument for voting $A$ and thus still a player who chooses rule 1 but votes for $B$ when rule 2 is implemented is behaving inconsistently.

Treatments 4 and 5 were implemented with 45 and 63 subjects, respectively, with random matching into groups of three in sessions with 12 to 24 participants. Otherwise, the same procedures were followed as for the other treatments.

\section{A.2. Results}

In Treatment 4 , we observe 62 instances where a subject had $\theta=-1$ and votes for rule 1 (18 percent of the cases where $\theta=-1$ ). In only 10 of these cases the rule is then chosen by default. In three cases the participants then make the inconsistent vote B. Thus, the number of observations where an unambiguous inconsistency across stages could in principle be detected is lower than expected and it is clearly too low to draw any clear inferences. ${ }^{27}$ There are two further reasons why the results from Treatment 4 were not as informative as we expected. First, it turned out that the participants exhibited substantial problems understanding the complicated instructions, which is the reason we then implemented the simpler Treatment 5. Second, due to a programming error, while the default rule choice was announced, it was not actually implemented. As a consequence, while at the voting stage subjects were informed in case of a default rule choice that the rule would be rule 2 , the actually implemented rule was sometimes instead rule 1, which subjects then learned at the end of the period. As a result, their confusion has likely grown (though surprisingly, nobody raised this issue during the experiment or in the post-experimental questionnaire). In any case, while Treatment 4 shows some evidence of a behavioral inconsistency across stages, its results should not be

\footnotetext{
${ }^{27}$ In particular, the 10 observations where an inconsistency could potentially be observed are concentrated on only six distinct participants, three of which then behave inconsistently exactly once.
} 
over-interpreted because the number of relevant observations was lower than expected and because many subjects were likely too confused. ${ }^{28}$

Treatment 5 provides clearer support to the view that there is an inconsistency across stages. In this Treatment, in 66 instances a subject with $\theta=-1$ suggests rule 1 (only about 14 percent of the cases where $\theta=-1$ ). In 18 of these cases, rule 2 is chosen instead. In only 4 of these cases, the subject then votes for $A$. Thus in 14 out of 18 cases where inconsistent behavior could occur, this is indeed observed. The 14 inconsistent choices are made by 10 distinct subjects, the four consistent choices by three distinct subjects, where one subject decides once consistently and once inconsistently. According to a binomial test, 14 out of 18 is significantly different from an equal distribution $(p<0.05)$. Taking into account repeat observations and that only 12 distinct subjects were ever in a situation where they could behave inconsistently, the 10 out of 12 who decide at least once inconsistently are also significantly different from an equal distribution $(p<0.05)$, whereas the nine out of 12 who are never consistent are not $(p>0.1)$.

Apparently, confusion also matters to a large degree in Treatment 5, because in 18 of the 48 cases where rule 1 is implemented after a subjects chose rule 1 , the subject votes for A, which reduces both own and total payoff if the vote becomes relevant. Still, the share of 10 out of 12 participants choosing inconsistently would require an even larger share of confused subjects. ${ }^{29}$ This indicates that indeed inconsistencies in the sense of subjects being more concerned about efficiency in the rule-choice stage than in the voting stage could be a relevant phenomenon. Given the low absolute number of these instances observed in Treatment 5 , this phenomenon should be subject to further research.

\footnotetext{
${ }^{28}$ Due to the fact that given the relatively low share of choices for rule 1 by construction only few instances of possible inconsistency could occur and because of the high degree of confusion, we decided not to re-run Treatment 4 with corrected software because this would very likely not have generated clearly interpretable data either. In post-experimental questionnaires, subjects in Treatment 4 mentioned more often than in the other treatments to have decided randomly. Some explain correctly how to decide selfishly in Treatment 4, but those that try to explain that they maximized expected total payoffs actually got it wrong because they explained that it would be good to always try to get outcome $A$. Hence there was indeed substantial confusion in Treatment 4.

${ }^{29}$ Just for illustration, ignoring the dependence between observations, 14 out of 18 and 18 out of 48 differ significantly according to a Fisher exact test $(p<0.01)$. Also in the post-experimental questionnaire of Treatment 5, some confusion is present in the sense that those who explain that they tried to maximize total payoffs do not show that they understand what it means to be pivotal for the different rules.
} 


\section{B. Modelling Efficiency Concerns}

In this section we study the optimal rule choice of an agent who cares about a weighted sum of all $2 n+1$ players' monetary payoffs in the game underlying Treatment 1 . Assume that this player has altruistic preferences captured by a utility function $u_{i}(\theta)=\gamma_{i} \theta_{i}+$ $\left(1-\gamma_{i}\right) \bar{\theta}_{-i}$, where $\bar{\theta}_{-i}=\frac{1}{2 n} \sum_{j \neq i} \theta_{j}$. We assume that agent $i$ expects that, for any rule, all players (including player $i$ himself) vote symmetrically and strictly selfishly in the second stage. ${ }^{30}$ That is, player $j$ votes for $A$ if $\theta_{j}>0$, votes for $B$ if $\theta_{j}<0$ and flips a fair coin if $\theta_{j}=0$. Given that the rule that player $i$ chooses only affects outcomes if that rule is chosen, we can treat the rule choice as if player $i$ could decide the rule for sure.

It is useful to first introduce some additional notation. Denote with $p_{k}^{+}$the probability that alternative $A$ is chosen if the rule is $k$ and player $i$ votes for $A$ and $p_{k}^{-}$the probability that $A$ is chosen if the rule is $k$ and $i$ votes for $B$.

Let $E_{k}^{+}$be the expected average payoff of the $2 n$ other players given rule $k$ and player $i$ voting for $A$ and $E_{k}^{-}$the expected average payoff of the other players given rule $k$ and player $i$ voting for $B$. Let $\omega_{j}$ be the expected average of the valuations of the other players conditional on at least $j$ among these players voting for $A$. Then $E_{k}^{+}=p_{k}^{+} \omega_{k-1}$ (because if at least $k-1$ of the other players vote for $A$ and player $i$ votes for $A$, then at least $k$ vote for $A$, so $A$ is chosen) and $E_{k}^{-}=p_{k}^{-} \omega_{k}$ (because if at least $k$ of the other players vote for $A$ and player $i$ votes for $B$, then at least $k$ vote for $A$, so $A$ is chosen). Moreover $E_{k}^{+}=p_{k}^{+} \omega_{k-1}=p_{k-1}^{-} \omega_{k-1}=E_{k-1}^{-}$for all $k \in\{2, . ., n\}$

Finally, denote by

$$
u_{i}^{+}\left(\theta_{i}, k\right):=\gamma p_{k}^{+} \theta_{i}+(1-\gamma) E_{k}^{+}
$$

the expected utility of player $i$ under rule $k$ conditional on all other players voting selfishly in the above defined sense and player $i$ voting for alternative A. We define $u_{i}^{-}\left(\theta_{i}, k\right)$ and $u_{i}^{0}\left(\theta_{i}, k\right)$, the expected utility when player $i$ votes for $B$ or flips a fair coin, respectively, in an analogous way.

Note that for all $k=1, . ., n$ the functions $u_{i}^{+}\left(\theta_{i}, k\right)$ and $u_{i}^{-}\left(\theta_{i}, k\right)$ are linear in the valuation $\theta_{i}$. Moreover $u_{i}^{+}\left(\theta_{i}, 1\right)=\gamma \theta_{i}$ because if player $i$ votes for A if rule 1 is chosen, $A$ is implemented for sure and hence nothing can be inferred about the valuations of the other players and hence their conditional expected valuation is 0 given the symmetry of the underlying distribution of valuations, $u_{i}^{-}\left(\theta_{i}, 2 n+1\right)=0$ (because in that case, $B$ is chosen for sure since unanimity would be required for $A$ but is prevented by $i$ ), $u_{i}^{+}(0, k)=(1-\gamma) E_{k}^{+} \geq 0$ because for rule 1 , nothing is learned about the other players'

\footnotetext{
${ }^{30}$ The optimality of the assumed second stage voting behavior will be discussed below.
} 
valuations from the implementation of $A$, whereas for the other rules it can be inferred that at least one of the other players has a positive valuation, and hence in that case the inequality is actually strict, and $u_{i}^{-}(0, k)=(1-\gamma) E_{k}^{-}>0$ because for any rule it can be inferred that at least one of the other players has a positive valuation.

It is a direct consequence of Proposition 2 in Schmitz and Tröger (2012) that the simple majority rule is optimal for agent $i$ when his valuation is zero. This, in combination with the strict monotonicity of the slopes of $u_{i}^{+}\left(\theta_{i}, k\right)$ and $u_{i}^{-}\left(\theta_{i}, k\right)\left(p_{k}^{+}\right.$and $\left.p_{k}^{-}\right)$in $k$ implies that rules $k>n+1(k<n+1)$ are suboptimal for agents with strictly positive (negative) valuations. Therefore, for agents with strictly positive (negative) valuations, it remains to compare all rules $k$ with $k \leq n+1(k \geq n+1)$. For $k \geq n+1, u_{i}^{-}(0, k)$ is strictly monotonously decreasing in $k$ and for $k \leq n+1, u_{i}^{+}(0, k)$ is strictly monotonously increasing in $k \cdot{ }^{31}$

This and the strict monotonicity of the slopes of $u_{i}^{+}\left(\theta_{i}, k\right)$ and $u_{i}^{-}\left(\theta_{i}, k\right)$ in $k$ implies that there are unique valuations $\theta_{k k^{\prime}}<0$ at which players are indifferent between any two rules $k \neq k^{\prime}$ with $k, k^{\prime} \geq n+1$. For the same reason, there are unique valuations $\theta_{k k^{\prime}}>0$ at which players are indifferent between any two rules $k \neq k^{\prime}$ with $k, k^{\prime} \leq n+1$. The following symmetry property is useful to prove our main result.

Lemma 1: For all $n \geq 1$, for all $j \in\{-n, \ldots, n\}$ and for all $\theta_{i}>0$ :

$$
u_{i}^{+}\left(\theta_{i}, n+1+j\right)-\frac{\gamma}{2} \theta_{i}=u_{i}^{-}\left(-\theta_{i}, n+1-j\right)-\frac{\gamma}{2}\left(-\theta_{i}\right)
$$

\section{Proof}

$$
\begin{gathered}
u_{i}^{+}\left(\theta_{i}, n+1+j\right)=p_{n+1+j}^{+}\left(\gamma \theta_{i}+(1-\gamma) \omega_{n+j}\right) \\
u_{i}^{-}\left(-\theta_{i}, n+1-j\right)=p_{n+1-j}^{-}\left(-\gamma \theta_{i}+(1-\gamma) \omega_{n+1-j}\right)
\end{gathered}
$$

Hence the claim is that

$$
p_{n+1+j}^{+}\left(\gamma \theta_{i}+(1-\gamma) \omega_{n+j}\right)=p_{n+1-j}^{-}\left(-\gamma \theta_{i}+(1-\gamma) \omega_{n+1-j}\right)+\gamma \theta_{i}
$$

\footnotetext{
${ }^{31}$ When $k \leq n+1, E_{k}^{+}$is increasing in $k$, because then cases where fewer than half of the other players have a positive valuation are dropped in the calculation of $E_{k}^{+}$. Similarly, when $k \geq n+1, E_{k}^{-}$ is decreasing in $k$, because then cases where more than half of the other players have a positive valuation are dropped in the calculation of $E_{k}^{-}$.
} 
We know that $p_{n+1+j}^{+}=1-p_{n+1-j}^{-}$(if the rule is $n+1+j$ and $i$ votes for $A$, then $A$ is implemented if at least $n+j$ others vote for $A$ and thus if they have a positive valuation, while if the rule is $n+1-j$ and $i$ votes for $B$, then $B$ is implemented if at least $2 n-(n+1-j)+1=n+j$ others vote for $B$ and thus if they have a negative valuation, and at least $n+j$ of the others having a positive valuation is equally likely as at least $n+j$ of them having a negative valuation). Hence the previous equality follows from

$$
p_{n+1+j}^{+} \omega_{n+j}=p_{n+1-j}^{-} \omega_{n+1-j} .
$$

Call $p_{m, 2 n}$ the probability that $m$ out of $2 n$ agents vote for $A$. We have, with $\bar{\theta}^{+}$ denoting the expected valuation conditional on voting for $A$ :

$$
\begin{aligned}
p_{n+1+j}^{+} \omega_{n+j} & =\sum_{m=n+j}^{2 n} p_{m, 2 n}(m-(2 n-m)) \bar{\theta}^{+} \\
& =2 \bar{\theta}^{+} \sum_{m=n+j}^{2 n} p_{m, 2 n}(m-n),
\end{aligned}
$$

and

$$
\begin{aligned}
p_{n+1-j}^{-} \omega_{n+1-j} & =\sum_{m=n+1-j}^{2 n} p_{m, 2 n}(m-(2 n-m)) \bar{\theta}^{+} \\
& =2 \bar{\theta}^{+} \sum_{m=n+1-j}^{2 n} p_{m, 2 n}(m-n) .
\end{aligned}
$$

Therefore, our claim can be rewritten as:

$$
\begin{aligned}
2 \bar{\theta}^{+} \sum_{m=n+j}^{2 n} p_{m, 2 n}(m-n) & =2 \bar{\theta}^{+} \sum_{m=n+1-j}^{2 n} p_{m, 2 n}(m-n) \\
\sum_{m=n+j}^{2 n} p_{m, 2 n}(m-n) & =\sum_{m=n+1-j}^{2 n} p_{m, 2 n}(m-n) \\
\sum_{m=n+1-j}^{n+j-1} p_{m, 2 n}(m-n) & =0
\end{aligned}
$$




$$
\begin{aligned}
\sum_{m=n+1-j}^{n-1} p_{m, 2 n}(m-n)+\sum_{m=n+1}^{n+j-1} p_{m, 2 n}(m-n) & =0 \\
\sum_{m=n+1}^{n+j-1} p_{m, 2 n}(m-n) & =\sum_{m=n+1-j}^{n-1} p_{m, 2 n}(n-m) .
\end{aligned}
$$

The last inequality holds because $p_{k, 2 n}=p_{2 n-k, 2 n}$.

Q.E.D.

Based on these preliminaries, we can prove the main result for the case of five players, i.e. $n=2$.

Proposition 1: For $n=2$, there exist $\underline{\theta}_{i}<\bar{\theta}_{i}<0$ such that player $i$ 's preferred rule is rule 5 for $\theta_{i}<\underline{\theta}_{i}$, rule 4 for $\underline{\theta}_{i}<\theta_{i}<\bar{\theta}_{i}$, rule 3 (majority rule) for $\bar{\theta}_{i}<\theta_{i}<-\bar{\theta}_{i}$, rule 2 for $-\bar{\theta}_{i}<\theta_{i}<-\underline{\theta}_{i}$, and rule 1 for $\theta_{i}>-\underline{\theta}_{i}$.

Proof Consider first $\theta_{i}<0$. If player $i$ chooses rule 5 , then her utility is 0 for sure (because she will vote for $B$ in the second stage). If she chooses rule 3 (majority rule) and her choice is implemented, then her expected utility is $p_{3}^{-}\left(\gamma \theta_{i}+(1-\gamma) \omega_{3}\right)$. So she is indifferent between rule 5 , which implements $B$ for sure and the majority rule, if $p_{3}^{-}\left(\gamma \theta_{i}+(1-\gamma) \omega_{3}\right)=0$ or $\theta_{i}=-\frac{(1-\gamma)}{\gamma} \omega_{3}$.

Now consider the intermediate rule 4. Player $i$ 's expected utility for $\theta_{i}=-\frac{(1-\gamma)}{\gamma} \omega_{3}$ and rule 4 is

$$
p_{4}^{-}\left(\gamma \theta_{i}+(1-\gamma) \omega_{4}\right)=p_{4}^{-}\left(-(1-\gamma) \omega_{3}+(1-\gamma) \omega_{4}\right)>0
$$

because $\omega_{4}>\omega_{3}$, since the expected average payoff $\omega_{k}$ increases strictly in $k$. Thus at the point where $i$ is indifferent between rule 5 and the majority rule, $i$ strictly prefers the intermediate rule.

The first part of the proposition follows from this and from (i) the linearity of all conditional utility functions (ii) the strict monotonicity of their slopes in $k$ and (iii) the fact that rule 3 strictly maximizes welfare conditional on $\theta_{i}=0$. The second part of the proposition follows from the symmetry property shown in Lemma 1, since, for all $\theta_{i}>0$ and $j \in\{0,2\}$,

$$
u_{i}^{+}\left(\theta_{i}, 2\right)=u_{i}^{+}\left(\theta_{i}, 3-j\right)
$$




$$
\begin{aligned}
& \Rightarrow u_{i}^{+}\left(\theta_{i}, 2\right)-\frac{\gamma}{2} \theta_{i}=u_{i}^{+}\left(\theta_{i}, 3-j\right)-\frac{\gamma}{2} \theta_{i} \\
& \Rightarrow \quad u_{i}^{-}\left(-\theta_{i}, 4\right)-\frac{\gamma}{2}\left(-\theta_{i}\right)=u_{i}^{-}\left(-\theta_{i}, 3+j\right)-\frac{\gamma}{2}\left(-\theta_{i}\right) \\
& \Rightarrow \quad u_{i}^{-}\left(-\theta_{i}, 4\right)=u_{i}^{-}\left(-\theta_{i}, 3+j\right) .
\end{aligned}
$$

Hence, indifference between rules 2 and 3 (2 and 1) for players with some type $\theta_{i}>0$ implies indifference of a player with type $-\theta_{i}$ between rules 4 and 3 ( 4 and 5). Q.E.D.

Hence, assuming selfish voting behavior, the optimal rule choice of efficiency-concerned subjects is monotonous and symmetric and, when the type space is large enough, it covers the entire set of available rules.

Moreover, one can easily show that, when player $i$ has no additional knowledge about other players' types, one can rule out that player $i$ prefers to vote non-selfishly under any voting rule that he has proposed himself. This follows because $u_{i}^{+}\left(\theta_{i}, k\right)=u_{i}^{-}\left(\theta_{i}, k-1\right)$ for all $k=2, . ., n$. Hence, if $\theta_{i}>0$ and $u_{i}^{+}\left(\theta_{i}, k\right) \geq u_{i}^{+}\left(\theta_{i}, k^{\prime}\right)$ for all $k^{\prime}=1, . ., 5$ then $u_{i}^{+}\left(\theta_{i}, k\right) \geq u_{i}^{-}\left(\theta_{i}, k^{\prime}-1\right)$ for all $k^{\prime}=2, . ., 5$. Furthermore, choosing rule 5 and voting for $B$ is worse than choosing rule $k$ and voting for $A$, i.e., if $\theta_{i}>0, u_{i}^{+}\left(\theta_{i}, k\right) \geq u_{i}^{-}\left(\theta_{i}, 5\right)=0$. The same type of argument can be made to rule out that players with negative valuations are better off by voting insincerely.

Selfish voting may instead not be optimal if another player's rule proposal has been successful. In such cases, player $i$ can infer something about other players' types from being pivotal in the voting stage. Moreover, player i may learn something about other player's expected valuations from the rule chosen in stage 1 . This is why non-selfish voting may be optimal for small positive or negative values of $i$ 's willingness to pay. Similarly, if an efficiency-concerned player expects other players to vote non-selfishly if certain rules are chosen, the rule choice may deviate from the one derived in Proposition 1.

\section{Regression Analysis for Treatment 1}

For a more thorough assessment of the rule choice in Treatment 1, we run ordered probit regressions of the rank number of the chosen rule on the valuation. ${ }^{32}$ We also run probit regressions for a dummy whether subjects are choosing a non-selfish rule because the key distinction appears to be between selfish and non-selfish rules. We run both the

${ }^{32}$ Ordered probit is more appropriate than OLS because the rule number cannot be considered as cardinal, given that one rule is the optimal one for a selfish player and the others vary rather gradually. 
ordered probit and probit regressions separately for negative and positive valuations, because this allows us to discriminate between the predictions of different preference types. Efficiency concerns would predict a roughly symmetric impact of the valuation both for negative and positive valuations, because the efficiency model presented in Appendix B is symmetric and the closer the valuation is to 0 , the more one should deviate from the selfish rule. ${ }^{33}$ In contrast, inequality aversion predicts that the valuation has no impact for $\theta_{i}<0$, because then the selfish rule should always be chosen, whereas for $\theta_{i}>0$, subjects should deviate more the smaller their valuation, as for the efficiencyconcerns model. The regressions again support the efficiency-concerns model. As can be seen in Table 5, the coefficient on the valuation is very similar for negative valuations and positive valuations in the ordered probit both without (column 1) and with (column 2) clustering of standard errors on the individual level as well as in the probit, both with individual-level random effects (column 3) and with clustering of standard errors on the individual level (column 4). ${ }^{34}$ Moreover, if there are differences in the significance levels, then in the direction of stronger significance for negative valuations, in contrast to the inequality aversion prediction.

\footnotetext{
${ }^{33}$ In theory, the effect should be exactly symmetric. However, since valuations are randomly assigned, valuations are typically not obtained by all subjects the same number of times and because subjects are likely to be heterogenous, this randomness will lead to some noise in the estimates, even if all subjects followed the efficiency concerns model consistently.

${ }^{34}$ It is in line with the efficiency concerns model that the coefficient in the probit changes sign, because for negative valuations, increasing valuation implies a lower absolute valuation and hence a higher willingness to choose non-selfishly.
} 


\begin{tabular}{cccccc}
\hline \hline \multicolumn{1}{c}{} & & $(1)$ & $(2)$ & $(3)$ & $(4)$ \\
\hline \multirow{4}{*}{$\theta_{i}<0$} & $\theta_{i}$ & $-.0729412^{* *}$ & $-.0729412^{*}$ & $.1728693^{* * *}$ & $.1100306^{* *}$ \\
& & $(.0316187)$ & $(.0429341)$ & $(.0574938)$ & $(.0454685)$ \\
& const. & & & $-.9027392^{* * *}$ & $-.4480241^{* *}$ \\
& Pseudo $R^{2}$ & 0.0102 & 0.0102 & $(.2980232)$ & $(.1837908)$ \\
\hline \hline \multirow{2}{*}{$\theta_{i}>0$} & $\theta_{i}$ & $-.0659364^{* *}$ & -.0659364 & $-.2054207^{* * *}$ & $-.077422^{*}$ \\
& & $(.0318391)$ & $(.0440765)$ & $(.0570684)$ & $(.0432327)$ \\
& & & & $-1.045793^{* * *}$ & $-.5213664^{* * *}$ \\
& const. & & & $(.3661205)$ & $(.1773873)$ \\
\hline \hline
\end{tabular}

Table 5: Regressions for chosen rule in Treatment 1. Columns (1) and (2) present order probit regressions for the rule chosen in stage 1 without and with clustering of standard errors at the individual level, respectively. Columns (3) and (4) present probit regressions of a dummy for not haven chosen the selfish rule in stage 1 with individual-level random effects and with clustering of standard errors at the individual level, respectively. The top part shows the results for valuations $\theta<0$, the bottom part for valuations $\theta>0$. Standard errors in parentheses. ${ }^{*}: p<0.1,{ }^{* *}: p<0.05,{ }^{* * *}: p<0.01$. 


\section{D: INSTRUCTIONS FOR TREATMENT 1}

(Note: in the instructions, rules were labelled in reverse order to that in the paper. We changed the order in the paper, because this makes the theory part easier to present.)

\section{INSTRUCTIONS}

You are now taking part in an experiment. The amount of money you earn depends on your choices and the choices of the other participants. It is therefore important that you understand the instructions. Please do not communicate with the other participants during the experiment. If you have any questions, please raise your hand and we will come to your seat.

All the information you provide will be treated anonymously.

You will begin the experiment with a starting budget of $9 €$. This amount can be increased or decreased depending on the choices and outcomes in one of the 15 rounds of the experiment. In each round, each player realizes a payoff which may be zero, positive or negative. In the end, one of the 15 rounds will be randomly selected and the payoff of this round will be added to/deducted from your starting budget. This yields your monetary earnings in the experiment. You should treat each round as if it were paid, since you do not know which round will determine your earnings. Your earnings will be paid out in cash after the experiment. The payout scheme is designed so that your earnings cannot be negative, that is, there is no risk that you will have to pay us. For each player, the minimum possible earnings of the entire experiment are $2 €$ and the maximum possible earnings of the entire experiment are $16 €$. Earnings will be treated confidentially.

Thank you for participating. 


\section{STRUCTURE OF THE EXPERIMENT}

In each round of the experiment you will be part of a group of 5 randomly selected participants. Your group has the opportunity to choose one of two alternatives (called A and B). Each group member's valuation of alternative B is zero. However, each group member may have a different valuation of alternative A. If the group decides in favour of alternative A, every group member has a payoff for this round equal to his or her individual valuation of alternative A (which is determined before the selection procedure starts). Otherwise, if the group decides in favour of alternative $B$, the payoff for this round is zero for each group member. Valuations of alternative A are determined anew in each round and the participant groups are randomly formed again each round.

The experiment is run through a computer program. Note that group members cannot recognize each other, so even if you encounter the same participant in different rounds, you will not be able to identify her or him.

The selection procedure consists of two stages. In the first stage, a voting rule is chosen, and in the second stage the group members vote which alternative is chosen according to this voting rule. We will now explain the two stages in detail.

\section{FIRST STAGE}

After the group is formed, all 5 group members propose a group decision rule, which determines how individual votes (for alternative $A$ or $B$ ) to be cast in the second stage are aggregated to form the group decision. The available rules are listed below. After all proposals for a group decision rule are collected, the group decision rule will be determined from the proposal of a single group member, who is chosen randomly. In other words, you do not know who in your group will determine the group decision rule. It may be the rule you proposed or the rule of any other group member, with equal probability. After the group decision rule is determined, it will be announced to all group members. None of the other proposals will be announced. Note that the group decision rule is of significance, since it can make it harder or easier for your preferred alternative to be chosen. 


\section{SECOND STAGE}

In the second stage, all group members vote for alternative $\mathbf{A}$ or $\mathbf{B}$, and the group decision automatically results from all individual votes according to the group decision rule determined in the first stage. The decision is announced and all players learn all five valuations from their group without being able to identify the other players.

\section{VALUATIONS}

In case alternative $A$ is chosen, all group members receive a payoff according to their individual valuation of alternative A. This means that if your valuation is positive, you may gain from a decision in favour of alternative A; if it is negative, you may lose. Your valuation of alternative $A$ is assigned to you randomly at the beginning of each round. Note that only you know its exact value. Similarly, you do not know the specific valuation of the others in your group. Individual valuations can have the following values: $-7 €,-3 €,-1 €,-0.5 €,-0.2 €,-0.1 €, 0 €, 0.1 €, 0.2 €, 0.5 €, 1 €, 3 €, 7 €$. Each valuation will be drawn with equal probability. Moreover, the valuations are distributed independently, that is, the value of your valuation is statistically independent of the valuation of the others. That means, whatever your valuation, this does not allow you to infer the valuations of the other group members. Similarly, valuations are drawn independently across periods. Hence your valuation in one round is not related in any way to your valuation in previous or later rounds.

Example: Assume your valuation in round 1 is $-3 €$, in round 2 it is $7 €$, and in round 3 it is $0 €$. If your group votes for alternative $A$ in each round, then your payoff (not necessarily earnings) is your valuation in that round. Thus, if round 1 were randomly chosen to count for your earnings, then your final earnings would be $6 €(=9 €-3 €)$. If round 2 were chosen, your earnings would be $16 €(=9 €+7 €)$, and for round 3 it would be $9 €$.

If the group chooses alternative $B$, everyone in the group receives a payoff of 0 in this round, that is, you will neither gain nor lose anything in that round independently of your valuation. Thus, if such a round is chosen to count for your final earnings, then you 
simply earn the starting budget, since you neither gained nor lost anything in the experiment.

Here is the structure of the experiment again in a short overview:

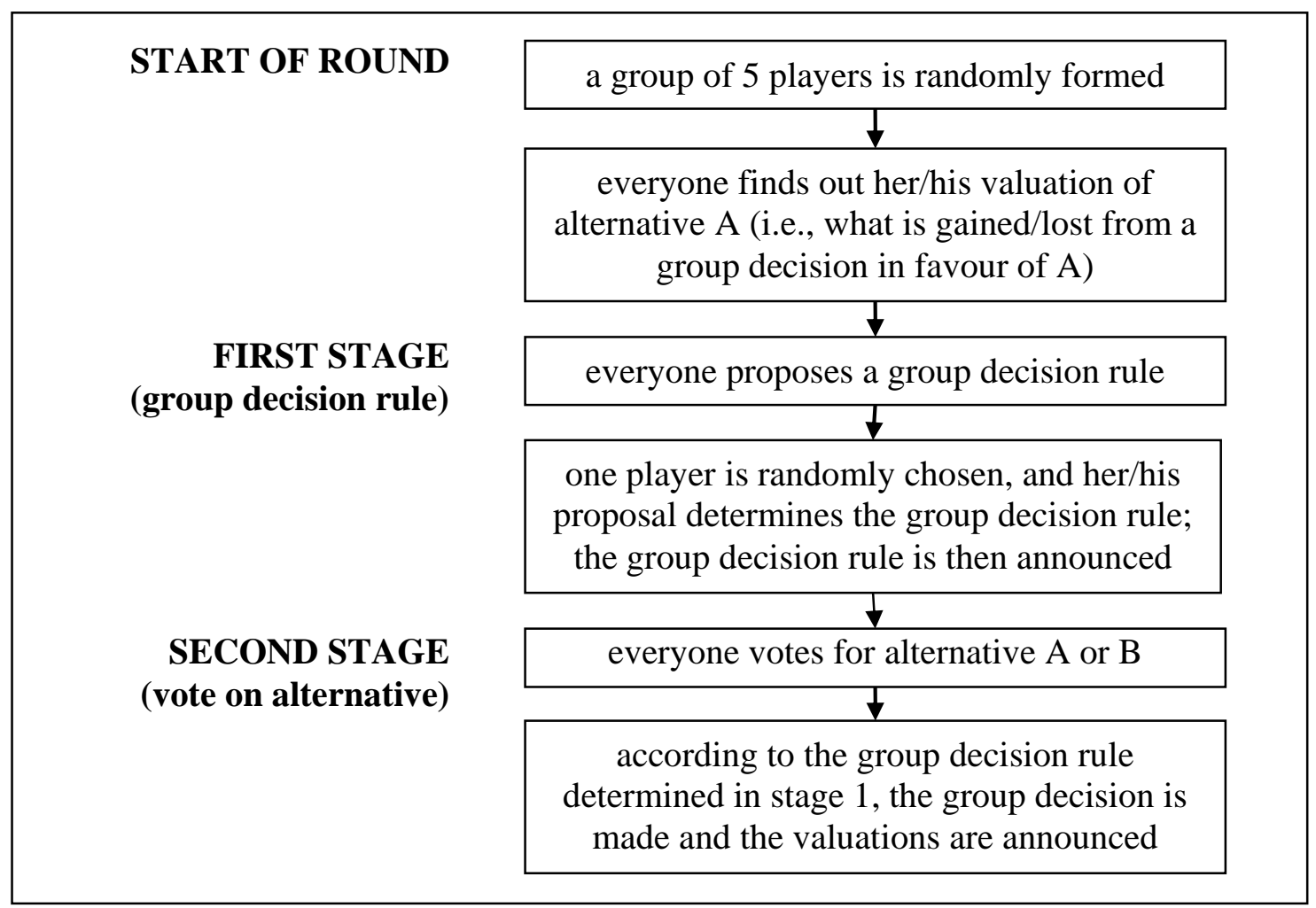

\section{AVAILABLE GROUP DECISION RULES}

In the first stage, each group member has to propose a group decision rule. Five rules are available:

I. 5 votes for alternative $A$ are required for $A$ to be chosen (unanimity for $\mathbf{A}$ ), thus at least 1 vote for alternative $\mathrm{B}$ is required for $\mathrm{B}$ to be chosen.

II. At least 4 votes for alternative $\mathrm{A}$ are required for $\mathrm{A}$ to be chosen (qualified majority for A), hence at least 2 votes for alternative $B$ are required for $B$ to be chosen. 
III. At least 3 votes are required for either A or B to be chosen (simple majority), that is, whichever has more votes wins.

IV. At least 2 votes for alternative $\mathrm{A}$ are required for $\mathrm{A}$ to be chosen, thus at least 4 votes for alternative B are required for B to be chosen (qualified majority for B).

V. At least 1 vote for alternative $A$ is required for $A$ to be chosen, thus 5 votes for alternative B are required for B to be chosen (unanimity for B).

Note that no one can abstain from voting, so, for example, only two votes for A automatically means three votes for B. 